IMA Journal of Applied Mathematics (2015) Page 1 of 34

doi:10.1093/imamat/xxx000

\title{
A shear-relaxation boundary layer near the pinned ends of a buckled elastic-walled tube
}

\author{
ROBERT J. WHITTAKER \\ School of Mathematics, University of East Anglia, Norwich Research Park, Norwich, NR4 7TJ
}

[Received on 28 January 2015]

\begin{abstract}
We consider small-amplitude deformations of a thin-walled elastic tube, which initially has a uniform elliptical cross-section and is subject to a large axial pre-stress. We derive a boundary-layer model for the deformations near an end of such a tube that is pinned to a rigid elliptical support. The model is appropriate in the limit in which $\mathscr{F} \equiv d^{2} F /\left[24 \pi a K\left(1-v^{2}\right)\right] \ll 1$, where $d$ is the wall thickness, $F$ is the axial tension that gives rise to the pre-stress, $2 \pi a$ is the tube circumference, $K$ is the bending stiffness of the tube wall, and $v$ is its Poisson ratio. In particular, the model takes into account in-plane shear forces arising because of geometrical constraints. These forces are asymptotically small outside the boundary layer, and so were not present in the previous tube-law model of Whittaker et al. (2010; Q. J. Mech. Appl. Math. 64(4), pp465-492).

Deformation profiles from the boundary-layer model are matched to solutions for the interior arising from the tube-law model of Whittaker et al. (2010). The net effect is to modify the previous tube-end boundary condition on the interior solution, from zero normal displacement to a Robin type condition. The predictions from the matched models compare favourably with full numerical simulations of the tube wall deformations. While the additional shear forces are only important in the boundary layer near the end, they can have a significant effect on the global solution when $\mathscr{F} \ll 1$.
\end{abstract}

Keywords: shell theory, boundary layers, in-plane shear, elastic-walled tubes

\section{Introduction}

Fluid flows through elastic-walled tubes are common in biological and industrial systems, and have received much attention through experimental, numerical and analytical studies. Comprehensive reviews of recent work can be found in Heil \& Jensen (2003), Grotberg \& Jensen (2004), and Heil \& Hazel (2011). Of particular interest are instabilities that can arise as a result of the kinematic and dynamic coupling between the interior fluid and the tube wall, in so-called 'fluid-structure interaction' problems.

Because of the complicated nature of even the simplest governing equations for the fluid and solid components, those wishing to undertake analytic studies have looked to simplify the equations to form reduced models. Examples of simplifications for the fluid flow include lumped parameter models (e.g. Bertram \& Pedley, 1982), the assumed flow-field used by Stewart et al. (2009) and the asymptotic analysis of Whittaker et al. (2010a).

For the solid mechanics of the tube wall, these reduced models often take the form of a 'tube law' - a relationship between the cross-sectional area $A$ of the tube and the transmural pressure $p=p_{\text {int }}-$ $p_{\text {ext }}$ at each axial position. Such laws have been derived both experimentally (e.g. Shapiro, 1977) and theoretically (e.g. Flaherty et al., 1972). Extensions to the basic $p=f(A)$ relationship have also been used (e.g. McClurken et al., 1981; Reyn, 1987), incorporating terms dependent on axial derivatives of $A$ (to capture axial bending or tension effects) and temporal derivatives of $A$ (to capture the effects of wall 
(a)

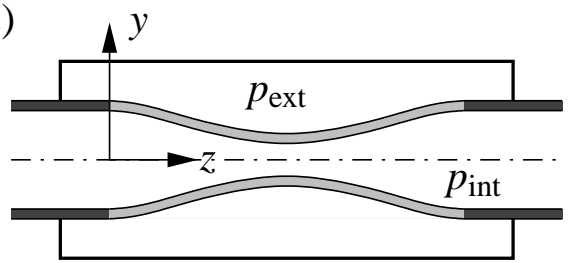

(b)

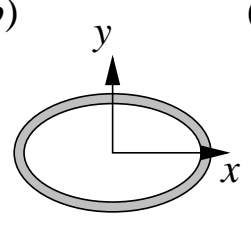

$(c)$

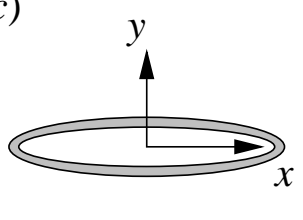

FIG. 1. A typical starling resistor setup, with an initially elliptical tube clamped between two rigid pipes and enclosed in a pressure chamber. (a) A view in the plane of the tube centre-line, showing the effect of a negative transmural pressure $p_{\text {int }}-p_{\text {ext }}$. (b) The undeformed elliptical cross-section of the tube, which matches the cross-section of the rigid pipes. (c) A typical deformed cross-section from the middle of the tube.

inertia).

The canonical experimental and computational setup for studying self-excited oscillations in elasticwalled tubes is the Starling resistor. In this setup, shown in figure 1, a length of elastic-walled tube is clamped between two rigid sections of tube. The flexible section is enclosed in a pressure chamber to allow the external pressure to be controlled. Flow is driven along the tubes by imposing the pressure and/or flux at the far upstream and downstream ends.

Because of the frequent use of this setup, it is important that any derived tube laws can reproduce the appropriate boundary conditions at the ends of the elastic section where it is clamped to the rigid sections. It is not obvious that this will always be possible, since tube laws are typically lower-order in the axial coordinate than the full 8th-order shell equations.

Such problems were seen to arise in Whittaker et al. (2010b). There, the authors presented a rational derivation of a tube-law model from the Kirchhoff-Love shell equations. The derived law involved terms proportional to the change in cross-sectional area and its second axial derivative (see (2.2) below). Solutions from the model generally compared well with numerical simulations of a Starling resistor under uniform and axially varying transmural pressures. However, in a certain region of parameter space, which corresponded to particularly thin tube walls (relative to other parameters), the agreement was less good. The problems were ascribed to the second-order tube-law not being able to satisfy the full set of four boundary conditions (three displacements and a rotation) at each point on the clamped circumference of tube. It was argued that the issue was not the neglect of axial bending, but rather the neglect of certain in-plane shear forces within the modelling.

In the present work, we address these issues with the model of Whittaker et al. (2010b), by deriving a boundary-layer description of the tube wall that re-introduces the neglected shear forces. The extra axial derivatives in the boundary-layer equations, mean that they are capable of matching between the interior tube-law solution and a more complete set of boundary conditions at the tube end.

This paper is organised as follows. In $\S 2$ we describe the work of Whittaker et al. (2010b) in more detail, and explain why a new boundary-layer model is required. In $\S 3$ we describe the tube geometry and the governing shell equations, and obtain the appropriate scalings for the boundary layer. In $\S 4$ the leading-order equations are derived, and we consider various solution methodologies in $\S 5$. It is found that the system depends crucially on a dimensionless parameter $\mathscr{F}$ (a rescaled axial tension), and that the boundary layer only has a significant effect on the solution when $\mathscr{F}$ is small. In $\S 6$, we present an asymptotic solution for the boundary layer for $\mathscr{F} \ll 1$. In $\S 7$ we derive effective boundary conditions on the interior solutions and show that the boundary layer imposes an $O\left(\mathscr{F}^{-1 / 2}\right)$ off-set in the boundary condition. In $\S 8$, we compute solutions for a tube subject to a uniform transmural pressure, and compare 


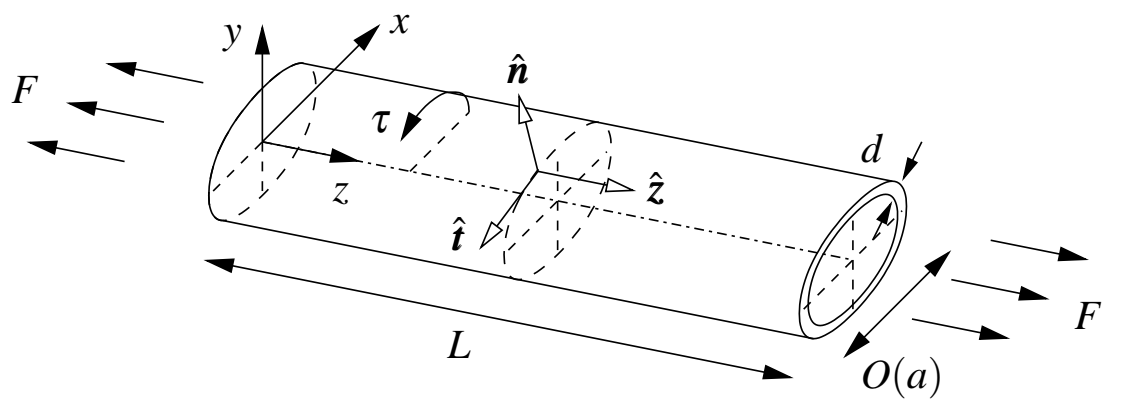

FIG. 2. The geometry and coordinates for the undeformed elastic tube. The tube has length $L$, wall thickness $d$ and an elliptical cross-section with diameters of $O(a)$. It is aligned with Cartesian axes $(x, y, z)$, and the two ends at $z=0$ and $z=L$ are fixed to rigid supports. The whole tube is subject to a pre-stress arising from an axial force $F$.

these with full numerical simulations. Finally, some concluding remarks are presented in $\S 9$.

\section{Mathematical Background}

The initially elliptical tube geometry considered by Whittaker et al. $(2010 b)$ is shown in figure 2 . The surface is parameterised by dimensionless Lagrangian coordinates $(\tau, \check{z})$ in the azimuthal and axial directions respectively. ( $\tau$ is an elliptical polar coordinate, and $\check{z}=z / L$ is scaled on the tube length $L$.) Deformations of the tube wall are then described by three dimensionless functions $(\check{\xi}, \check{\eta}, \check{\zeta})$ of $\tau$ and $\check{z}$, which give the displacements in the normal $\hat{n}$, azimuthal $\hat{\boldsymbol{t}}$ and axial $\hat{z}$ directions respectively.

In the tube-law model developed in Whittaker et al. (2010b), the full Kirchhoff-Love shell equations for the tube wall are reduced to a single second-order ODE in ž. First, asymptotic methods are used (based on a regime of small-amplitude long-wavelength deformations of a thin-walled tube) to obtain a single PDE for $\check{\eta}(\tau, \check{z})$. The PDE has the form

$$
\tilde{F} \frac{\partial^{2}}{\partial \check{z}^{2}} \mathscr{L}_{2}(\check{\eta})-\mathscr{L}_{6}(\check{\eta})=-\tilde{P}(\check{z})
$$

where the $\mathscr{L}_{n}$ are $n$ th-order linear differential operators in $\tau, \tilde{F}$ is the dimensionless axial tension applied to the tube, and $\tilde{P}$ is the dimensionless applied transmural pressure. The axial order of the system is reduced from 8 to 2 , because of the asymptotic neglect of the forces that result from axial bending and in-plane shearing. The normal and axial displacement fields $\breve{\xi}$ and $\breve{\zeta}$ have been eliminated by using the asymptotic result that, geometrically, there is negligible shear and negligible azimuthal stretching. (These geometric constraints allow $\breve{\xi}$ and $\breve{\zeta}$ to be written in terms of $\check{\eta}$ at leading order.)

Whittaker et al. (2010b) then further simplify the PDE (2.1) using the observation that a single azimuthal mode dominates the deformations. They approximate $\check{\eta} \approx e_{1}(\check{z}) \sin (2 \tau)$ and show that, at leading order, the amplitude $e_{1}(\check{z})$ is proportional to the dimensionless change $\alpha(\check{z})$ in the cross-sectional area of the tube. An ODE was thus obtained for $\alpha$ as a function of $\check{z}$ :

$$
k_{2} \tilde{F} \frac{\partial^{2} \alpha}{\partial \check{z}^{2}}-k_{0} \alpha=-\tilde{P}(\check{z}),
$$

where $k_{0}$ and $k_{2}$ are numerically determined constants related to the shapes of the initial cross-section and the deformation mode. 

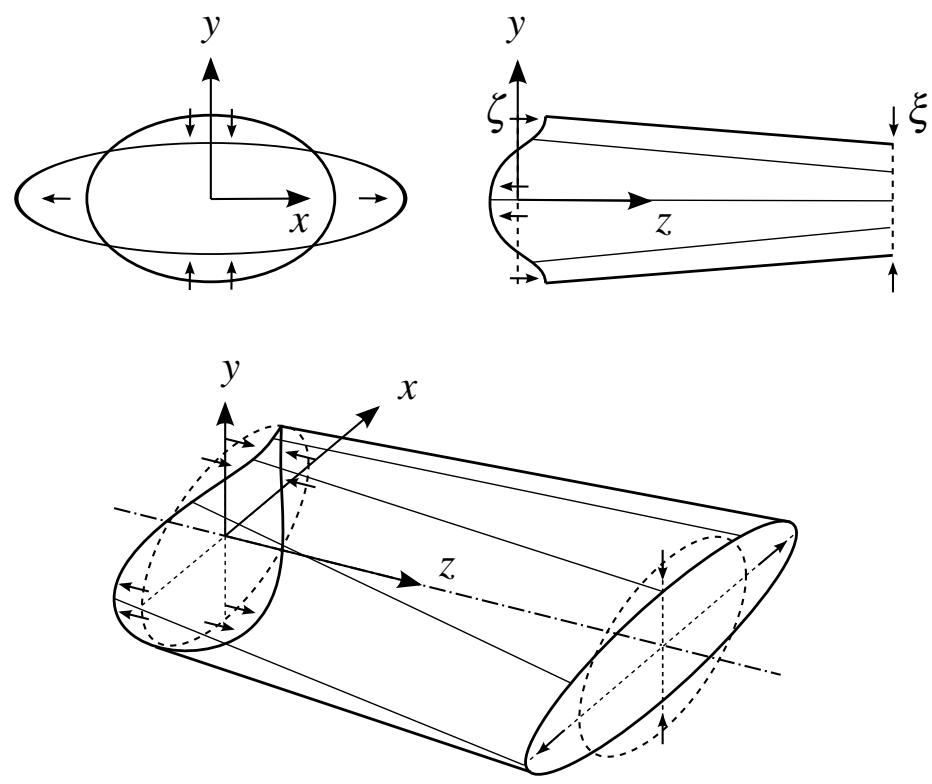

FIG. 3. Exaggerated sketches showing a deformation of the tube wall near one end under the asymptotic model of Whittaker et al. $(2010 \mathrm{~b})$. In the absence of in-plane shearing and axial bending, a typical deformation in the bulk of the tube forces a non-zero axial displacement $\zeta$ and a non-zero surface gradient $\partial \xi / \partial z$ at the tube end.

With the reduced axial order, it is impossible for a general solution of (2.2) to satisfy the full set of boundary conditions that we should impose on the shell at the ends of the tube. For example, if the flexible section is bonded to rigid tubes at each end (e.g. in a Starling resistor setup) then we should impose the canonical 'clamped' conditions, where position and gradient of the shell are specified at the boundary (i.e. $\check{\xi}=\check{\eta}=\check{\zeta}=\partial \check{\xi} / \partial \check{z}=0$ ). However, the tube-law model (2.2) is only second-order in $\check{z}$, and so allows only one quantity, such as $\alpha$ (or equivalently the azimuthal displacements $\check{\eta}$ ), to be set set to zero at each end of the tube, e.g.

$$
\alpha=0 \quad \text { at } \quad \check{z}=0,1
$$

The assumption of negligible azimuthal stretching means the normal displacements $\breve{\xi}$ are then also set to zero at $\check{z}=0,1$ in the model. However, the axial displacements $\check{\zeta}$ and surface gradient $\partial \check{\xi} / \partial \check{z}$ (which are also determined by the solution) will not, in general, turn out to be zero at $\check{z}=0,1$.

The inability to set the gradient $\partial \breve{\xi} / \partial \check{z}=0$ arises from the neglect of the fourth-order axial derivatives that correspond to axial bending forces. The inability to set the axial displacement $\breve{\zeta}$ is more subtle, and arises from the neglect of the forces associated with in-plane shearing of the shell.

As it stands, the model can only produce solutions with non-zero axial displacements and gradients at the tube ends, as shown in figure 3. The question is then whether or not these solutions are good approximations to the solution of the full shell equations with a full set of 'clamped' boundary conditions imposed.

In Whittaker et al. (2010b), this issue was investigated by solving the full shell equations numerically, with three different sets of tube-end boundary conditions. In addition to the 'clamped' boundary condition, two other conditions were tested: the canonical 'pinned' conditions, and a non-standard 
condition that was termed 'sliding'. The 'pinned' conditions fix the position of the shell boundary $(\breve{\xi}=\check{\eta}=\breve{\zeta}=0$ ), but instead of fixing the gradient, they fix the end of the shell to be torque-free. The solution from the model is not precisely torque-free, but this should be much closer to the behaviour of the model. The 'sliding' conditions fix the normal and azimuthal displacements $(\breve{\xi}=\breve{\eta}=0)$, but the axial displacements and gradient are set by the imposition of zero axial stress perturbation and zero torque respectively. Again, this is not precisely what the solution of the model attains, but should be much closer.

The numerical solutions presented by Whittaker et al. (2010b) show that in the regime of interest, results from the clamped and pinned boundary conditions are very similar, suggesting that the inability to set the gradient does not significantly affect the model. However, the difference between the clamped and sliding boundary conditions is very significant in some (though not all) regions of the parameter space. This suggests that the inability to set the axial displacements can significantly affect the model.

In this work, we therefore consider how the tube-law model of Whittaker et al. (2010b) can be amended to give an additional degree of freedom that would allow the axial displacements to be set to zero at the ends. As with other singular perturbations, we expect that a suitable re-scaling of the governing equations will reveal a boundary-layer region near the end, which is governed by a higherorder equation. This will allow one or more additional conditions to be set at the boundary, while still being able to match to a solution from the previous model in the bulk interior.

\section{Mathematical setup and scaling analysis}

\subsection{Problem description}

As in Whittaker et al. (2010b), we consider an elastic-walled tube which is initially an axially uniform elliptical cylinder with length $L$, circumference $2 \pi a$, and wall thickness $d$, as shown in figure 2 . The ellipticity of the tube is set by a parameter $\sigma_{0}$ so that the major-minor axis ratio is given by coth $\sigma_{0}$. The tube wall has bending stiffness $K$ and Poisson ratio $v$. In its initial elliptical configuration, the tube is subject to a uniform axial pre-stress, due to an axial tension $F$. The two ends of the tube are pinned to rigid elliptical supports. We wish to consider deformations induced by an applied transmural pressure $p$ (possibly axially varying), with dimensional scale $P$.

We recall the following dimensionless parameters (and their scales) from Whittaker et al. (2010b):

$$
\ell=\frac{L}{a} \gg 1, \quad \delta=\frac{d}{a} \ll 1, \quad \tilde{F}=\frac{a F}{2 \pi K \ell^{2}}=O(1), \quad \varepsilon=\frac{a^{3} P}{K} \ll 1 .
$$

The parameters $\ell$ and $\delta$ are aspect ratios, assumed to be large and small respectively for a long thinwalled tube. $\tilde{F}$ is a dimensionless measure of the axial tension, taken to be $O(1)$ so that the restoring force from the axial tension has the same magnitude as the restoring force from azimuthal bending of the tube wall. Finally $\varepsilon a$ is a scale-estimate for the size of the deformations induced by the external pressure $P$, chosen to be small compared with the typical tube radius $a$.

Material points on the tube wall are described by a pair of dimensionless coordinates $(\tau, z)$ defined so that the position of the un-deformed tube wall is is given by

$$
\overline{\boldsymbol{r}}(\tau, z)=a\left(\begin{array}{c}
c \cosh \sigma_{0} \cos \tau \\
c \sinh \sigma_{0} \sin \tau \\
z
\end{array}\right)
$$

The dimensionless coordinates therefore lie in the ranges $\tau \in(0,2 \pi)$ and $z \in(0, \ell)$. The dimensionless 
constant $c$ is given by

$$
c=\frac{\pi \operatorname{sech} \sigma_{0}}{2 \operatorname{Ee}\left(\operatorname{sech} \sigma_{0}\right)},
$$

so that the circumference of the tube is precisely $2 \pi a$. (Here $\operatorname{Ee}(k)$ is the complete elliptic integral of the second kind.) The deformed position of the tube wall is then written as a vector function $\boldsymbol{r}(\tau, z)$ of the material coordinates $(\tau, z)$. The pinned boundary conditions at the tube ends imply that

$$
\boldsymbol{r}(\tau, z)=\overline{\boldsymbol{r}}(\tau, z) \quad \text { at } \quad z=0, \ell .
$$

We also define unit vectors $\hat{\boldsymbol{t}}$ and $\hat{\boldsymbol{z}}$ aligned respectively with the $\tau$ and $z$ coordinates in the undeformed surface. The unit normal to the undeformed surface is then given by $\hat{\boldsymbol{n}}=\hat{\boldsymbol{t}} \times \hat{\boldsymbol{z}}$. These vectors are shown in figure 2 .

For later convenience, we introduce the scale factor

$$
h(\tau)=c\left(\frac{1}{2} \cosh 2 \sigma_{0}-\frac{1}{2} \cos 2 \tau\right)^{1 / 2},
$$

associated with the $\tau$ coordinate, and the dimensionless azimuthal curvature $\bar{B}(\tau)$ in the undeformed state. In Appendix A we show that

$$
\bar{B} \equiv \hat{\boldsymbol{n}} \cdot \frac{1}{h} \frac{\partial \hat{\boldsymbol{t}}}{\partial \tau}=-\frac{c^{2} \sinh 2 \sigma_{0}}{2 h^{3}} .
$$

For the description of the bulk behaviour of the tube, Whittaker et al. (2010b) use an axial coordinate scaled on the axial length $L$. We use $\check{z}=z / \ell$ to represent this coordinate.

\subsection{Kirchhoff-Love shell equations}

We adopt the same coordinate system and governing equations to describe the elastic tube wall as in Whittaker et al. $(2010 b)$. We work with dimensional Lagrangian coordinates $\left(x^{1}, x^{2}\right)$ to parameterise the shell mid-plane $\boldsymbol{r}\left(x^{1}, x^{2}\right)$, chosen so that in the undeformed configuration we have

$$
x^{1}=a h \tau, \quad x^{2}=a z .
$$

The metric tensor $a_{\alpha \beta}$ and curvature tensor $b_{\alpha \beta}$ are then defined by

$$
a_{\alpha \beta}=\frac{\partial \boldsymbol{r}}{\partial x^{\alpha}} \cdot \frac{\partial \boldsymbol{r}}{\partial x^{\beta}}, \quad b_{\alpha \beta}=\boldsymbol{a}_{3} \cdot \frac{\partial^{2} \boldsymbol{r}}{\partial x^{\alpha} \partial x^{\beta}},
$$

where $a_{3}$ is the unit normal to the (deformed) shell surface.

In the absence of tangential body forces and wall inertia, the Kirchhoff-Love shell equations used by Whittaker et al. $(2010 b)$ are

$$
\begin{aligned}
\nabla_{\alpha} \nabla_{\beta} M^{\alpha \beta}+N^{\alpha \beta} b_{\alpha \beta} & =-p, \\
\nabla_{\beta} N^{\beta 1}-b_{\gamma}^{1} \nabla_{\beta} M^{\beta \gamma} & =0, \\
\nabla_{\beta} N^{\beta 2}-b_{\gamma}^{2} \nabla_{\beta} M^{\beta \gamma} & =0 .
\end{aligned}
$$

where $N^{\alpha \beta}$ is the in-plane stress tensor, $M^{\alpha \beta}$ is the bending moment tensor, and $\nabla_{\alpha}$ is the covariant derivative in the direction $x^{\alpha}$. 
The deformation of the wall material is characterised by in-plane strain and bending strain tensors

$$
\gamma_{\alpha \beta}=\frac{1}{2}\left(a_{\alpha \beta}-\bar{a}_{\alpha \beta}\right), \quad \kappa_{\alpha \beta}=-b_{\alpha \beta}+\bar{b}_{\alpha \beta}+2 \bar{b}_{\alpha}^{\delta} \gamma_{\delta \beta},
$$

where over-bars denote the values of quantities in the undeformed configuration.

Linear constitutive laws relate the stress and stress moment resultants $N^{\alpha \beta}$ and $M^{\alpha \beta}$ to the strains $\gamma_{\alpha \beta}$ and $\kappa_{\alpha \beta}$ as follows (Flügge, 1972, $\left.\S 9.4\right){ }^{1}$

$$
\begin{gathered}
N^{\alpha \beta}=\delta_{2}^{\alpha} \delta_{2}^{\beta} \frac{F}{2 \pi a}+D\left[(1-v) \gamma^{\alpha \beta}+v \gamma_{\lambda}^{\lambda} a^{\alpha \beta}\right] \\
+K\left\{\frac{(1-v)}{2}\left[2 a^{\beta \delta} b^{\alpha \gamma}+a^{\beta \gamma} b^{\alpha \gamma}+a^{\alpha \delta} b^{\beta \gamma}-b_{\lambda}^{\lambda}\left(a^{\alpha \delta} a^{\beta \gamma}+a^{\alpha \gamma} a^{\beta \delta}\right)\right]\right. \\
\left.+v\left[a^{\alpha \beta} b^{\gamma \delta}+a^{\gamma \delta} b^{\alpha \beta}-a^{\alpha \beta} a^{\gamma \delta} b_{\lambda}^{\lambda}\right]\right\} \kappa_{\gamma \delta}, \\
M^{\alpha \beta}=K\left[-(1-v)\left(b_{\gamma}^{\alpha} \gamma^{\gamma \beta}-b_{\lambda}^{\lambda} \gamma^{\alpha \beta}\right)-v\left(b^{\alpha \beta}-b_{\lambda}^{\lambda} a^{\alpha \beta}\right) \gamma_{\mu}^{\mu}\right. \\
\left.+\frac{1}{2}(1-v)\left(\kappa^{\alpha \beta}+\kappa^{\beta \alpha}\right)+v a^{\alpha \beta} \kappa_{\lambda}^{\lambda}\right]
\end{gathered}
$$

where the extensional stiffness $D$ is related to the bending stiffness $K$ by

$$
D=\frac{12 K}{a^{2} \delta^{2}}
$$

The constitutive laws (3.13) and (3.14) arise from inserting the plane-stress form of Hooke's law into the definitions of $N^{\alpha \beta}$ and $M^{\alpha \beta}$, rewriting the resulting equations in terms of $\gamma_{\alpha \beta}$ and $\kappa_{\alpha \beta}$, and neglecting some higher-order terms in $\delta$.

\subsection{Scaling analysis}

We perform a similar analysis of the magnitudes of the terms in the equilibrium shell equations to that in Whittaker et al. (2010b). The key difference here is that in boundary layer, there will be no knowledge of the overall tube-length, so this can have no effect on the axial length scale. We therefore must determine the appropriate axial length scale from the equations themselves.

In order to match on to the bulk interior solution from Whittaker et al. (2010b) as we leave the boundary layer, the normal and azimuthal displacements must be growing linearly in $z$, while the axial displacement must become independent of $z$. The relationship between these quantities must also be such that the shear and azimuthal stretching (both assumed to be negligible in the original model) become negligible as we leave the boundary layer.

These matching requirements suggest that all three displacements (normal, azimuthal, and axial) should scale with $\varepsilon a / \ell$, and that the axial length scale of the boundary layer should be comparable with the $O(a)$ tube diameter. Using these scalings, we can recompile the tables in Whittaker et al. (2010b) that show the dominant contributions to the three stress-balance equations from different physical effects. ${ }^{2}$

\footnotetext{
${ }^{1}$ Some signs in (3.2) differ from those in Flügge (1972). This is due to our opposing sign conventions on $\kappa_{\alpha \beta}$ and $M^{\alpha \beta}$, and later because of a sign error on the $\left(\kappa_{\alpha \beta}+\kappa_{\beta \alpha}\right)$ term in Flügge's expression for $M^{\alpha \beta}$.

${ }^{2}$ The tables are omitted here for the sake of brevity. The details merely serve to confirm that the choice of scalings will lead to a sensible physical balance, and show which terms we expect to be present. The derivations below in $\S 3.4$ include all the necessary calculations to obtain the leading-order governing equations.
} 
In Whittaker et al. (2010b), we had that the physical effects that were present in the leading-order balances were the transmural pressure, azimuthal bending, and the combined effects of the pre-stress and axial curvature. With the shorter axial length we consider here, we find that the scale estimates for the 'pre-stress and axial curvature' terms are larger, owing to the axial derivatives they contain. These terms are now asymptotically larger than the transmural pressure, and so the latter will have negligible effect in the boundary layer. The other terms which may be present in the leading-order balance come from stretching effects. Unlike in the bulk interior region, where the solution was constrained to have limited stretching and shearing, these effects will now be significant.

\subsection{Dimensionless displacements and stresses}

Motivated by the scalings above, we write the displacements of the wall from the undeformed configuration as

$$
\boldsymbol{r}-\overline{\boldsymbol{r}}=\frac{\varepsilon a}{\ell}\left(\frac{1}{h(\tau)}[\xi(\tau, z) \hat{\boldsymbol{n}}+\eta(\tau, z) \hat{\boldsymbol{t}}]+\zeta(\tau, z) \hat{z}\right) .
$$

in terms of dimensionless functions $(\xi, \eta, \zeta)$, which we expect to be $O(1)$ in the boundary layer.

Whittaker et al. (2010b) used a different scaling for the displacements, appropriate for the bulk of the tube. They also needed to include two differently scaled functions for the axial displacements. We denote their displacement functions by $\left(\check{\xi}, \check{\eta}, \check{\zeta}, \check{\zeta}_{a}\right)$ and find that

$$
\xi=\ell \check{\xi}, \quad \eta=\ell \check{\eta}, \quad \zeta=\check{\zeta}+\delta^{2} \ell^{2} \check{\zeta}_{a} .
$$

In Appendix A we use the representation (3.16) to derive expressions for $a^{\alpha \beta}, b^{\alpha \beta}, \gamma^{\alpha \beta}$ and $\kappa^{\alpha \beta}$ in terms of $(\xi, \eta, \zeta)$. Inserting these expressions into the constitutive laws (3.13)-(3.14), we can derive leading-order expressions for the stress and moment tensors, for $\varepsilon \ll 1, \delta \ll 1$ and $\ell \gg 1$. We find that the in-plane stress tensor $N^{\alpha \beta}$ is given asymptotically by

$$
N^{\alpha \beta}=\frac{K}{a^{2}}\left(\begin{array}{cc}
0 & 0 \\
0 & \ell^{2} \tilde{F}
\end{array}\right)+\frac{\varepsilon K}{a^{2} \delta^{2} \ell}\left(\begin{array}{cc}
\tilde{N} & \tilde{S} \\
\tilde{S} & \tilde{\Sigma}
\end{array}\right)+O(\varepsilon K / \ell)
$$

where

$$
\tilde{N}=12\left(-\frac{\bar{B} \xi}{h}+\frac{1}{h} \frac{\partial}{\partial \tau}\left(\frac{\eta}{h}\right)+v \frac{\partial \zeta}{\partial z}\right),
$$

is the leading-order azimuthal hoop stress,

$$
\tilde{S}=\frac{12(1-v)}{2 h}\left(\frac{\partial \eta}{\partial z}+\frac{\partial \zeta}{\partial \tau}\right)
$$

is the leading-order in-plane shear stress, and

$$
\tilde{\Sigma}=12\left(\frac{\partial \zeta}{\partial z}+v\left(-\frac{\bar{B} \xi}{h}+\frac{1}{h} \frac{\partial}{\partial \tau}\left(\frac{\eta}{h}\right)\right)\right) .
$$

is the leading order axial stress.

We also find that the leading order bending stress scales as

$$
M^{\alpha \beta}=O\left(\frac{\varepsilon K}{a \ell}\right)
$$


which is sufficiently small so as not contribute to the leading-order equilibrium equations. We therefore do not consider $M^{\alpha \beta}$ any further here.

In addition to the expressions for $N^{\alpha \beta}$ and $M^{\alpha \beta}$, in order to consider the governing equations (3.9)(3.11) we need to be able to interpret the curvature tensor $b_{\alpha \beta}$ and the covariant derivatives $\nabla_{\alpha}$.

From Appendix A, we have that the curvature tensor $b_{\alpha \beta}$ has the following representation:

$$
b_{\alpha \beta}=\frac{\bar{B}}{a}\left(\begin{array}{cc}
1 & 0 \\
0 & 0
\end{array}\right)+\frac{\varepsilon}{a \ell}\left(\begin{array}{cc}
O(1) & O(1) \\
O(1) & \frac{1}{h} \frac{\partial^{2} \xi}{\partial z^{2}}
\end{array}\right) \text {. }
$$

The components represented by the $O(1)$ scales do not contribute to our model at leading order, and hence their precise forms are not required.

Finally, we need to be able to write the covariant derivatives $\nabla_{\alpha}$ in terms of partial derivatives. As in Whittaker et al. (2010b), we have

$$
\nabla_{\alpha}=\frac{\partial}{\partial x^{\alpha}}+O(\varepsilon) \Rightarrow \nabla_{1}=\frac{1}{a h} \frac{\partial}{\partial \tau}+O(\varepsilon), \quad \nabla_{2}=\frac{1}{a} \frac{\partial}{\partial z}+O(\varepsilon)
$$

and the only place we need to worry about the correction terms is when they are applied to the large axial pre-stress in $N^{22}$. The relevant expression is

$$
\nabla_{\alpha} N^{\alpha \beta}=\frac{\partial N^{\alpha \beta}}{\partial x^{\alpha}}+\Gamma_{\gamma \alpha}^{\alpha} N^{\gamma \beta}+\Gamma_{\gamma \alpha}^{\beta} N^{\alpha \gamma}
$$

where $\Gamma_{\beta \gamma}^{\alpha}$ is the Christoffel symbol. We show in Appendix A that the components we need are

$$
\Gamma_{21}^{1}=\frac{1}{a} \frac{\partial \gamma_{11}}{\partial z}, \quad \Gamma_{22}^{1}=\frac{\varepsilon}{a \ell h} \frac{\partial^{2} \eta}{\partial z^{2}}, \quad \Gamma_{22}^{2}=\frac{\varepsilon}{a \ell} \frac{\partial^{2} \zeta}{\partial z^{2}}
$$

\section{The shear-relaxation boundary layer}

We now construct the leading-order equations governing the displacements within the boundary layer near $z=0$, when $\varepsilon \ll 1, \delta \ll 1$, and $\ell \gg 1$. We also derive the appropriate boundary conditions for a pinned boundary at $z=0$, and the matching conditions for matching with an interior solution in the bulk of the tube.

\subsection{Leading-order equilibrium equations}

We take the equilibrium equations (3.9)-(3.11), and substitute in the representations (3.18) and (3.23) for $N^{\alpha \beta}$ and $b_{\alpha \beta}$, note the scaling estimate (3.22) for $M^{\alpha \beta}$, and use the expressions (3.24)-(3.26) for the covariant derivatives. We then look for the leading order contributions in each of the three directions.

In the normal direction, the dominant balance in (3.9) comprises the interaction between the azimuthal hoop $\tilde{N}$ stress and the base state curvature $\bar{B}$, together with the interaction between the axial pre-stress $\tilde{F}$ and the axial curvature $\partial^{2} \xi / \partial z^{2}$. (These forces are greater than the applied transmural pressure, so the latter does not contribute at leading order.) At leading order, we obtain

$$
N^{11} b_{11}+N^{22} b_{22}=0 \quad \Rightarrow \quad \frac{\bar{B}}{\delta^{2}} \tilde{N}+\frac{\tilde{F} \ell^{2}}{h} \frac{\partial^{2} \xi}{\partial z^{2}}=0
$$


In the azimuthal direction, the dominant balance in (3.10) comprises derivatives of the azimuthal hoop stress $\tilde{N}$ and in-plane shear stress $\tilde{S}$, together with the interaction between the axial pre-stress $\tilde{F}$ and the in-plane curvature $\partial^{2} \eta / \partial z^{2}$. At leading order, we obtain

$$
\frac{\partial N^{11}}{\partial x^{1}}+\frac{\partial N^{21}}{\partial x^{2}}+\Gamma_{22}^{1} N^{22}=0 \quad \Rightarrow \quad \frac{1}{\delta^{2}} \frac{\partial \tilde{N}}{\partial \tau}+\frac{h}{\delta^{2}} \frac{\partial \tilde{S}}{\partial z}+\ell^{2} \tilde{F} \frac{\partial^{2} \eta}{\partial z^{2}}=0 .
$$

In the axial direction, the dominant balance in (3.11) comprises derivatives of the shear stress $\tilde{S}$ and the axial stress $\tilde{\Sigma}$, together with interactions of various curvatures with the axial pre-stress $\tilde{F}$. At leading order, we obtain

$$
\begin{aligned}
& \frac{\partial N^{12}}{\partial x^{1}}+\frac{\partial N^{22}}{\partial x^{2}}+\Gamma_{21}^{1} N^{22}+2 \Gamma_{22}^{2} N^{22}=0 \\
& \Rightarrow \quad \frac{1}{\delta^{2} h} \frac{\partial \tilde{S}}{\partial \tau}+\frac{1}{\delta^{2}} \frac{\partial \tilde{\Sigma}}{\partial z}+\ell^{2} \tilde{F} \frac{\partial}{\partial z}\left(-\bar{B} \frac{\xi}{h}+\frac{1}{h} \frac{\partial}{\partial \tau}\left(\frac{\eta}{h}\right)\right)+2 \ell^{2} \tilde{F} \frac{\partial^{2} \zeta}{\partial z^{2}}=0 .
\end{aligned}
$$

\subsection{Governing equations for the displacements}

Apart from the Poisson ratio, $v$, the only other parameter combination present in the leading-order equations (4.1)-(4.3) is $\delta^{2} \ell^{2} \tilde{F}$. We therefore introduce a new parameter

$$
\mathscr{F}=\frac{\delta^{2} \ell^{2} \tilde{F}}{12\left(1-v^{2}\right)}=\frac{d^{2} F}{24 \pi a K\left(1-v^{2}\right)} .
$$

An additional factor of $12\left(1-v^{2}\right)$ has been included, because this will simplify the analysis of the limit $\mathscr{F} \rightarrow 0$ (which turns out to be the important regime to consider) below.

Physically, $\mathscr{F}$ represents the ratio of the stresses that arise from the axial tension, to those that arise from in-plane stretching and shearing, both evaluated on the length scale $a$ of the tube diameter. The former can be estimated as the size of the pre-stress times the second spatial derivative of the deformations, giving $F /(2 \pi a)(\varepsilon a / \ell)\left(1 / a^{2}\right)=\varepsilon F /\left(2 \pi a^{2} \ell\right)$. The latter can be estimated as the extensional stiffness times the deformation gradient times the base-state curvature, giving $D(\varepsilon / \ell)(1 / a)=12 \varepsilon K /\left(a d^{2} \ell\right)$.

We now substitute the expressions (3.19)-(3.21) for the stresses $\tilde{N}, \tilde{S}$ and $\tilde{\Sigma}$ into the leading-order equilibrium equations (4.1)-(4.3). We obtain

$$
\begin{array}{r}
\bar{B}\left(-\bar{B} \frac{\xi}{h}+\frac{1}{h} \frac{\partial}{\partial \tau}\left(\frac{\eta}{h}\right)+v \frac{\partial \zeta}{\partial z}\right)+\mathscr{F}\left(1-v^{2}\right) \frac{\partial^{2} \xi}{\partial z^{2}}=0, \\
\frac{\partial}{\partial \tau}\left(-\bar{B} \frac{\xi}{h}+\frac{1}{h} \frac{\partial}{\partial \tau}\left(\frac{\eta}{h}\right)+v \frac{\partial \zeta}{\partial z}\right)+\frac{(1-v)}{2} \frac{\partial}{\partial z}\left(\frac{\partial \eta}{\partial z}+\frac{\partial \zeta}{\partial \tau}\right)+\mathscr{F}\left(1-v^{2}\right) \frac{\partial^{2} \eta}{\partial z^{2}}=0, \\
\frac{(1-v)}{2 h} \frac{\partial}{\partial \tau}\left[\frac{1}{h}\left(\frac{\partial \eta}{\partial z}+\frac{\partial \zeta}{\partial \tau}\right)\right]+\frac{\partial}{\partial z}\left[\frac{\partial \zeta}{\partial z}+v\left(-\bar{B} \frac{\xi}{h}+\frac{1}{h} \frac{\partial}{\partial \tau}\left(\frac{\eta}{h}\right)\right)\right] \\
+\mathscr{F}\left(1-v^{2}\right)\left[\frac{\partial}{\partial z}\left(-\bar{B} \frac{\xi}{h}+\frac{1}{h} \frac{\partial}{\partial \tau}\left(\frac{\eta}{h}\right)\right)+2 \frac{\partial^{2} \zeta}{\partial z^{2}}\right]=0 .
\end{array}
$$

\subsection{Boundary and matching conditions}

At $z=0$, we have the tube end with its pinned conditions (3.4), which specify no displacements. We therefore must have

$$
\xi=\eta=\zeta=0 \quad \text { on } \quad z=0
$$


As $z \rightarrow \infty$ the solution must match on to an interior solution that satisfies the tube law developed in Whittaker et al. (2010b). In particular, this means that the in-plane shear $\tilde{S}$ and the azimuthal hoop stress $\tilde{N}$ must become negligible on the scales introduced here. Examining the normal and azimuthal force-balance equations (4.1) and (4.2), we see that this implies

$$
\frac{\partial^{2} \xi}{\partial z^{2}} \rightarrow 0 \quad \text { and } \quad \frac{\partial^{2} \eta}{\partial z^{2}} \rightarrow 0 \quad \text { as } \quad z \rightarrow \infty
$$

The axial stretching $\partial \zeta / \partial z$ must also become negligible as we leave the boundary layer, because the axial length scale in the boundary layer is much shorter than the axial length scale in the interior. Therefore we require

$$
\frac{\partial \zeta}{\partial z} \rightarrow 0 \quad \text { as } \quad z \rightarrow \infty
$$

Hence $\zeta(\tau, z)$ must tend to a function of $\tau$ only. We define $f(\tau)$ to be this function. Then, using the fact that $\tilde{N}, \tilde{S} \rightarrow 0,(3.19)$ and (3.20) imply that we must have

$$
\begin{aligned}
& \xi \sim-z \frac{1}{\bar{B}} \frac{\partial}{\partial \tau}\left(\frac{f^{\prime}(\tau)}{h}\right)+\frac{1}{\bar{B}} \frac{\partial}{\partial \tau}\left(\frac{g(\tau)}{h}\right), \\
& \eta \sim-z f^{\prime}(\tau)+g(\tau), \\
& \zeta \sim f(\tau)
\end{aligned}
$$

as $z \rightarrow \infty$, for some functions $f(\tau)$ and $g(\tau)$. The functions $f$ and $g$ are arbitrary, in the sense that any $f$ and $g$ in (4.11)-(4.13) give an appropriate behaviour in $\xi, \eta$ and $\zeta$ as $\tau \rightarrow \infty$, which can be matched to an interior solution.

The matching condition itself then requires that the displacements $\xi, \eta$ and $\zeta$ match, which will yield conditions on the interior solution involving $f(\tau)$ and $g(\tau)$. The full interior system in Whittaker et al. $(2010 b)$ is the PDE (2.1) for the single variable $\check{\eta}$, which is second-order in $z$. We therefore need to match $\check{\eta}$ and its axial derivative. Using (3.17) and (4.12), we obtain the conditions

$$
\check{\eta}=\frac{1}{\ell} g(\tau) \quad \text { and } \quad \frac{\partial \check{\eta}}{\partial \check{z}}=-f^{\prime}(\tau) \quad \text { at } \quad \check{z}=0
$$

The equations used to recover $\breve{\xi}$ and $\breve{\zeta}$ from $\check{\eta}$ in the interior yield solutions that are already consistent with the asymptotic forms (4.11) and (4.13). Therefore no additional matching conditions are required for $\breve{\xi}$ and $\breve{\zeta}$.

\subsection{Combined Boundary-layer and interior problem}

The boundary-layer system (4.5)-(4.7) for $\xi, \eta, \zeta$ and the interior system (2.1) for $\check{\eta}$ are to be solved subject to the boundary conditions (4.8) at $z=0$, the required asymptotic behaviours (4.11)-(4.13) as $z \rightarrow \infty$, and the matching conditions (4.14). A second boundary layer would appear at the other end of the tube, which would be matched in a corresponding manner. It is verified in Appendix B that we have the correct number of boundary and matching constraints for the number of degrees of freedom in the combined system. 


\section{Methods of Solution}

The full boundary-layer-interior system cannot be solved analytically, so we would need to resort to a numerical solution. As a linear elliptic system of PDEs this should not present too much difficulty. However, in Whittaker et al. (2010b), the interior PDE (2.1) was reduced to an ODE (2.2) by assuming the dominance of a single azimuthal mode. It would be convenient if boundary-layer system here could be solved separately from the interior PDE, and then the boundary-layer solution used to determine effective boundary conditions on the interior that can be applied to the ODE system.

\subsection{Limit of a circular cross section}

At this point, it is informative to consider the limit as the elliptical cross-section becomes circular, i.e. $\sigma_{0} \rightarrow \infty$. In this limit, we have $h(\tau) \rightarrow 1$ and $\bar{B}(\tau) \rightarrow-1$, which simplifies the equations (4.5)-(4.7) somewhat. It is then possible to obtain an analytic solution to the boundary-layer system using a Fourier expansion in $\tau$.

The details are presented in Appendix C. For the $n$th Fourier mode, the asymptotic behaviour (4.11)(4.13) has $g(\tau)=z_{n}^{*} f^{\prime}(\tau)$ for some eigenvalue $z_{n}^{*}$. The functions $f(\tau)$ and $g(\tau)$ are simply Fourier modes, and analytic expressions for the $z_{n}^{*}$ are obtained as part of the solution. We find that $z_{n}^{*}$ decreases as the mode number $n$ increases. The boundary condition on the interior system that arises through the matching can then be expressed in terms of the known eigenfunctions $f(\tau)$ and eigenvalues $z_{n}^{*}$.

\subsection{Numerical eigenvalue problem}

Based on the outcome for the case of a circular cross section, we propose looking for solutions to the boundary-layer system in which $g(\tau)=z^{*} f^{\prime}(\tau)$ in (4.11)-(4.13) for some constant $z^{*}$. This leads to the following eigenvalue problem for the boundary-layer system: (4.5)-(4.7) are to be solved for $\xi, \eta, \zeta$, subject to:

$$
\xi=\eta=\zeta=0 \quad \text { at } \quad z=0
$$

and

$$
\begin{aligned}
& \xi \sim-\frac{1}{\bar{B}} \frac{\partial}{\partial \tau}\left(\frac{f_{n}^{\prime}(\tau)}{h}\right)\left(z-z_{n}^{*}\right), \\
& \eta \sim-f_{n}^{\prime}(\tau)\left(z-z_{n}^{*}\right), \\
& \zeta \sim f_{n}(\tau),
\end{aligned}
$$

as $z \rightarrow \infty$, where $z_{n}^{*}$ is an eigenvalue and $f_{n}(\tau)$ its corresponding eigenfunction, both to be found as part of the solution. By analogy with the circular case, we would expect to find a set of eigenfunctions corresponding to different azimuthal modes, with the eigenvalues $z_{n}^{*}$ decreasing as the azimuthal mode number increases.

Once the eigenvalue problem has been solved, the boundary conditions on the interior solution $\check{\eta}$ would then be

$$
\check{\eta}=\frac{1}{\ell} \sum_{n=1}^{\infty} G_{n} z_{n}^{*} f_{n}^{\prime}(\tau) \quad \text { and } \quad \frac{\partial \check{\eta}}{\partial \check{z}}=-\sum_{n=1}^{\infty} G_{n} f_{n}^{\prime}(\tau) \quad \text { at } \quad \check{z}=0 \text {, }
$$

for some set of amplitudes $G_{n}$. By writing $\check{\eta}$ as a sum over the eigenfunction derivatives: $\check{\eta}=\sum_{n} b_{n}(z) f_{n}^{\prime}(\tau)$, we can eliminate the $G_{n}$ from (5.5) to obtain a Robin boundary condition on the amplitude of each component:

$$
b_{n}(0)+\frac{z_{n}^{*}}{\ell} b_{n}^{\prime}(0)=0 .
$$


Since $\ell \gg 1$, we can see from (5.6) that unless $z_{n}^{*}$ is large for some $n$, the applied boundary conditions on the interior solution will be little different from specifying $\check{\eta}=0$. As the lower modes are likely to be excited more by the interior solution, and $z_{n}^{*}$ is likely to decrease as $n$ increases, we will need $z_{0}^{*}$ to be large to obtain any significant deviation from the original $\check{\eta}=0$ Dirichlet condition.

In the circular case, we see in Appendix $\mathrm{C}$ that $z_{n}^{*} \sim \mathscr{F}-1 / 2$ for both $\mathscr{F} \ll 1$ and $\mathscr{F} \gg 1$. We shall therefore concentrate on the $\mathscr{F} \ll 1$ regime. We expect this to be the only regime in which $z_{0}^{*}$ will be large, and hence the only regime in which the boundary layer will have a significant effect on the interior solution.

It turns out that we can make significant analytic progress in the $\mathscr{F} \ll 1$ asymptotic regime, the details of which are provided below in $\S 6$. Since this work provides the boundary conditions we need in the only regime of interest, there is little to be gained from solving the full numerical eigenvalue problem (4.5)-(4.7) and (5.1)-(5.4) posed here. We therefore did not attempt this computation.

\section{Asymptotic solution for $\mathscr{F} \ll 1$}

The limiting form of the boundary layer as $\mathscr{F} \rightarrow 0$ is quite subtle. As we shall see below, the boundary layer splits into two distinct sub-layers. The different stresses $\{\tilde{N}, \tilde{S}, \tilde{\Sigma}\}$ have different magnitudes from each other in each layer, and from themselves between the layers. This means that while the displacements $\{\xi, \eta, \zeta\}$ may be $O(1)$ everywhere, certain combinations of them are smaller in one or other layer. This results in some leading-order cancellations between certain terms in the equations. To avoid a leading-order degeneracy, it is necessary to capture the first-order corrections to some of these cancellations.

The easiest way to do this is to re-cast the problem in terms of new variables which do not suffer from leading-order cancellations. The natural choice is to use the physical stress variables $\{\tilde{N}, \tilde{S}, \tilde{\Sigma}\}$. The change of variables is carried out in Appendix D, where the new governing equations (A.44)-(A.46) are obtained.

The deformations considered in Whittaker et al. (2010b) were $\pi$-periodic in $\tau$ and had a definite parity: $\breve{\xi}$ and $\breve{\zeta}$ being even functions of $\tau$, while $\check{\eta}$ was odd. We therefore restrict attention here to solutions with the same symmetries; namely solutions for $\{\tilde{N}, \tilde{\Sigma}, \tilde{S}\}$ that are $\pi$-periodic in $\tau$, with $\tilde{N}$ and $\tilde{\Sigma}$ even and $\tilde{S}$ odd.

It is convenient to use (A.46) to eliminate $\tilde{S}$ from (A.44) and (A.45). Doing so, the boundary-layer system (4.5)-(4.7) is transformed to a pair of coupled equations for $\tilde{N}$ and $\tilde{\Sigma}$ :

$$
\begin{aligned}
-\bar{B}^{2} \tilde{N}-v \mathscr{F} \tilde{N}_{\tau \tau}+\mathscr{F} \tilde{\Sigma}_{\tau \tau}+\mathscr{F}(1+2 \mathscr{F}(1+v)(1-2 v)) \tilde{N}_{z z} \\
+\mathscr{F}(2(1+v)-v+2 \mathscr{F}(1+v)(2-v)) \tilde{\Sigma}_{z z}=0 \\
-(1+v \mathscr{F}) \tilde{N}_{\tau \tau}+\mathscr{F} \tilde{\Sigma}_{\tau \tau}+\mathscr{F}(1-2 v)(1+2 \mathscr{F}(1+v)) \tilde{N}_{z z} \\
+(1+2 \mathscr{F}(1+v))(1+\mathscr{F}(2-v)) \tilde{\Sigma}_{z z}=0 .
\end{aligned}
$$

As in Appendix D, we use a subscript $z$ to represent the usual partial derivative with respect to $z$, but a subscript $\tau$ to represent the operator

$$
\frac{1}{h(\tau)} \frac{\partial}{\partial \tau}
$$

The boundary conditions for (6.1) and (6.2) come from (A.48) and (A.49) and are

$$
\tilde{N}-v \tilde{\Sigma}=0 \quad \text { on } \quad z=0, \quad \tilde{N}, \tilde{\Sigma} \rightarrow 0 \quad \text { as } \quad z \rightarrow \infty .
$$


Once a solution for $\tilde{N}$ and $\tilde{\Sigma}$ has been found, the shear stress $\tilde{S}$ can be recovered using (A.46) and the condition that $\tilde{S}$ must be odd in $\tau$. Then the original displacement variables $\{\xi, \eta, \zeta\}$ can be calculated using (A.37)-(A.39).

Examining the system (6.1)-(6.4), it can be seen that the limit $\mathscr{F} \rightarrow 0$ is singular in two different ways. Naïvely setting $\mathscr{F}=0$ in (6.1) and (6.2), the equations reduce to

$$
\tilde{N}=0, \quad \tilde{\Sigma}_{z z}=0 .
$$

These equations are lower order in $z$, and also their solution is incapable of satisfying the boundary conditions (6.4) as $z \rightarrow \infty$. We therefore expect to find an inner boundary layer near $z=0$, and an outer layer as $z \rightarrow \infty$. In the inner boundary layer, a shorter axial length scale allows some of the $z$ derivatives in (6.1) to contribute, thus returning the system to being fourth-order in $z$. In the outer boundary layer, a longer axial length scale means that $\tilde{\Sigma}_{z z}$ will be small enough to be balanced by some of the other terms in (6.2), thus allowing decaying modes as $z \rightarrow \infty$.

\subsection{The inner boundary layer}

The following re-scalings are found to give a consistent description of an inner boundary layer, with a shorter axial length scale:

$$
\begin{aligned}
z & =\mathscr{F}^{1 / 2} \hat{z}, \\
\tilde{N} & =\mathscr{F}^{1 / 2} \hat{N}=\mathscr{F}^{1 / 2}\left(\hat{N}^{(0)}+\mathscr{F} \hat{N}^{(1)}+\ldots\right), \\
\tilde{\Sigma} & =\mathscr{F}^{1 / 2} \hat{\Sigma}=\mathscr{F}^{1 / 2}\left(\hat{\Sigma}^{(0)}+\mathscr{F} \hat{\Sigma}^{(1)}+\ldots\right), \\
\tilde{S} & =\hat{S}=\left(\hat{S}^{(0)}+\mathscr{F} \hat{S}^{(1)}+\ldots\right) .
\end{aligned}
$$

The magnitudes of $\tilde{N}$ and $\tilde{\Sigma}$ must be the same to allow the boundary condition (6.4) to be satisfied at $z=0$. (The $O\left(\mathscr{F}^{1 / 2}\right)$ scale for these is set a posteriori so that the asymptotic forms $(6.47)-(6.49)$ for the displacements have the appropriate magnitude to match to the bulk interior solution.) The axial length scale is then chosen so that the $\partial^{2} / \partial z^{2}$ terms re-enter in (6.1). Finally, the magnitude of $\tilde{S}$ is set by a scaling analysis of (A.46).

We substitute the scaled variables (6.6)-(6.9) into the governing equations (6.1)-(6.2) and take the limit $\mathscr{F} \rightarrow 0$. The leading-order equations that result are

$$
\begin{aligned}
-\bar{B}^{2} \hat{N}^{(0)}+\hat{N}_{\hat{z} \hat{z}}^{(0)}+(2+v) \hat{\Sigma}_{\hat{z} \hat{z}}^{(0)} & =0, \\
\hat{\Sigma}_{\hat{z} \hat{z}}^{(0)} & =0,
\end{aligned}
$$

and the equation (A.46) to recover $\hat{S}$ becomes

$$
\hat{S}_{\tau}^{(0)}=-\hat{\Sigma}_{\hat{z}}^{(0)} .
$$

Solving (6.10)-(6.12) and applying the boundary condition (6.4) at $z=0$, we obtain

$$
\begin{aligned}
\hat{N}^{(0)} & =n_{-}(\tau) \mathrm{e}^{-|\bar{B}(\tau)| \hat{z}}+n_{+}(\tau) \mathrm{e}^{+|\bar{B}(\tau)| \hat{z},} \\
\hat{\Sigma}^{(0)} & =\frac{1}{v}\left[n_{-}(\tau)+n_{+}(\tau)\right]-s_{\tau}(\tau) \hat{z}, \\
\hat{S}^{(0)} & =s(\tau),
\end{aligned}
$$

where $n_{ \pm}$and $s$ are arbitrary functions of $\tau$. 


\subsection{The outer boundary layer}

The following re-scalings are found to give a consistent description of an outer boundary layer, with a longer axial length scale:

$$
\begin{aligned}
& z=\mathscr{F}^{-1 / 2} \breve{z}, \\
& \tilde{N}=\mathscr{F}^{3 / 2} \breve{N}=\mathscr{F}^{3 / 2}\left(\breve{N}^{(0)}+\mathscr{F} \breve{N}^{(1)}+\ldots\right), \\
& \tilde{\Sigma}=\mathscr{F}^{1 / 2} \breve{\Sigma}=\mathscr{F}^{1 / 2}\left(\breve{\Sigma}^{(0)}+\mathscr{F} \breve{\Sigma}^{(1)}+\ldots\right) \\
& \tilde{S}=\mathscr{F} \breve{S}=\mathscr{F}\left(\breve{S}^{(0)}+\mathscr{F} \breve{S}^{(1)}+\ldots\right)
\end{aligned}
$$

The axial length scale is chosen to allow the largest $\tilde{\Sigma}$ terms to re-enter in (6.2). The magnitude of $\tilde{\Sigma}$ must be $O\left(\mathscr{F}^{1 / 2}\right)$ to allow matching with a non-trivial inner layer. The magnitude of $\tilde{N}$ is then reduced to allow the $\tilde{\Sigma}_{\tau \tau}$ term to balance the $\tilde{N}$ term in (6.1). Finally, the magnitude of $\tilde{S}$ is set by a scaling analysis of (A.46).

We substitute the scaled variables (6.16)-(6.19) into the governing equations (6.1)-(6.2) and take the limit $\mathscr{F} \rightarrow 0$. The leading-order equations that result are

$$
\begin{aligned}
-\bar{B}^{2} \breve{N}^{(0)}+\breve{\Sigma}_{\tau \tau}^{(0)} & =0, \\
-\breve{N}_{\tau \tau}^{(0)}+\breve{\Sigma}_{\tau \tau}^{(0)}+\breve{\Sigma}_{\breve{z}}^{(0)} & =0,
\end{aligned}
$$

and the equation (A.46) to recover $\hat{S}$ becomes

$$
\breve{S}_{\tau}^{(0)}=-\breve{\Sigma}_{\breve{z}}^{(0)}
$$

On eliminating $\breve{N}$ between (6.20) and (6.21), we obtain

$$
\breve{\Sigma}_{\breve{z} \breve{z}}^{(0)}-\mathscr{L}\left(\breve{\Sigma}^{(0)}\right)=0
$$

where $\mathscr{L}$ is the operator

$$
\mathscr{L} \equiv \frac{1}{h} \frac{\partial}{\partial \tau} \frac{1}{h} \frac{\partial}{\partial \tau}\left(\frac{1}{\bar{B}^{2} h} \frac{\partial}{\partial \tau} \frac{1}{h} \frac{\partial}{\partial \tau}-1\right)
$$

It can be shown that $\mathscr{L}$ is self-adjoint with respect to the inner product $\langle f, g\rangle=\int_{0}^{2 \pi} f g h \mathrm{~d} \tau$. We find that $\mathscr{L}$ has a countably infinite set of even $\pi$-periodic eigenfunctions $Y_{n}(\tau)$ with distinct positive eigenvalues $\mu_{n}^{2}$. Without loss of generality, we take $0=\mu_{0}<\mu_{1}<\mu_{2}<\mu_{3} \ldots$. The eigenfunctions are orthogonal with respect to the inner product, and we see that $Y_{0}(\tau)$ is constant.

In the limit $\sigma_{0} \rightarrow \infty$ (which corresponds to a circular cross-section; see Appendix C) we have $\mathscr{L} Y \equiv$ $Y^{\prime \prime \prime \prime}-Y^{\prime \prime}$, and it is easy to show that

$$
Y_{n}(\tau)=\cos (2 n \tau), \quad \mu_{n}=2 n\left(4 n^{2}+1\right)^{1 / 2} .
$$

For finite $\sigma_{0}$, the modes and eigenvalues can only be found numerically. Plots showing the lowest-order modes for $\sigma_{0}=0.6$, and the $n=1$ modes for a range of $\sigma_{0}$, can be found in figure 4 . The corresponding eigenvalues $\mu_{n}$ are shown in figure 5 . 
(a)

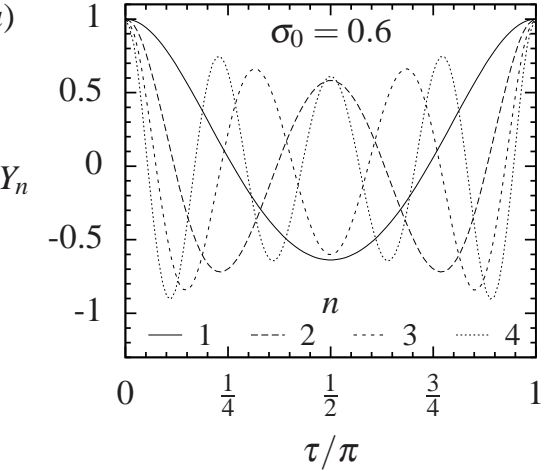

(b)

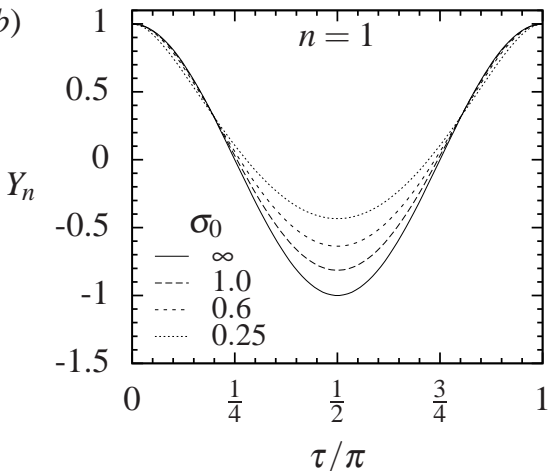

FIG. 4. Numerically computed eigenfunctions $Y_{n}(\tau)$ of the operator $\mathscr{L}$, as defined in (6.24). (a) The first few modes for $\sigma_{0}=0.6$. (b) The $n=1$ mode for a range of different ellipticities $\sigma_{0}$. In both parts, we have used the arbitrary normalisation $Y_{n}(0)=1$. The corresponding eigenvalues are shown in figure 5.

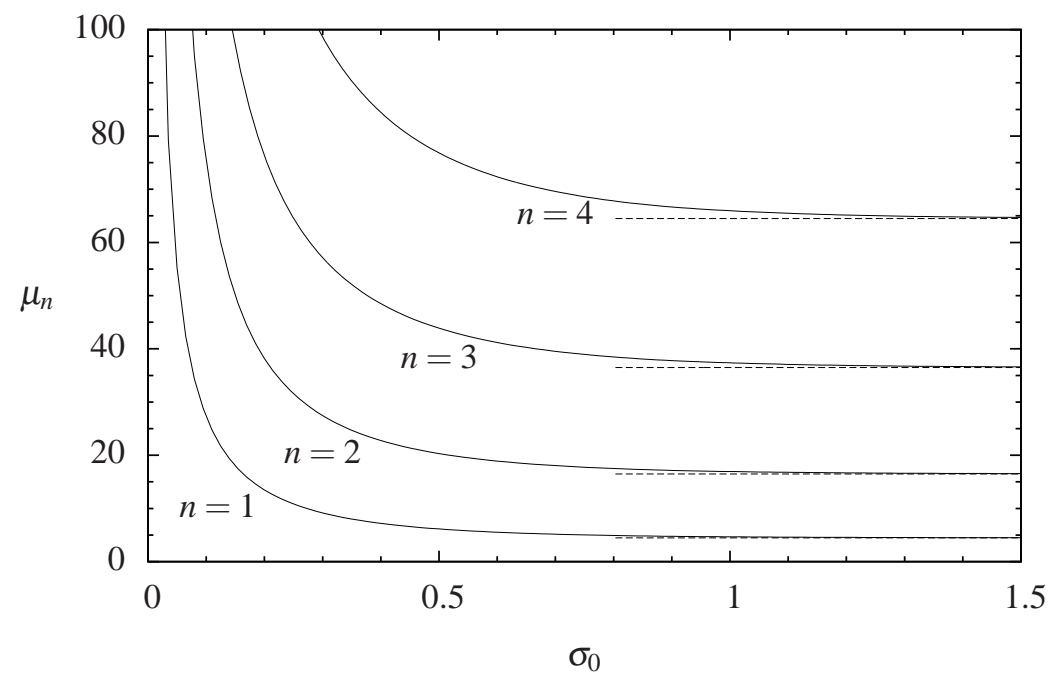

FIG. 5. Numerically computed eigenvalues $\mu_{n}$ of the operator $\mathscr{L}$ (as defined in (6.24)) as functions of ellipticity $\sigma_{0}$. The corresponding eigenfunctions are shown in figure 4. The dashed lines give the asymptotic solutions (6.25) for $\sigma_{0} \gg 1$ (the limit of a circular cross-section). 
Using separation of variables, the general solution to $(6.23)$ for $\breve{\Sigma}^{(0)}$ with the required symmetries can be written in terms of $Y_{n}(\tau)$ and $\mu_{n}$ as

$$
\Sigma^{(0)}=A_{0}+B_{0} \breve{z}+\sum_{n=1}^{\infty} Y_{n}(\tau)\left(A_{n} \mathrm{e}^{\mu_{n} \breve{z}}+B_{n} \mathrm{e}^{-\mu_{n} \breve{z}}\right),
$$

where the $A_{n}$ and $B_{n}$ are arbitrary constants.

Using (6.20) and (6.22) we then find

$$
\begin{aligned}
\breve{N}^{(0)}= & \sum_{n=1}^{\infty} \frac{1}{\bar{B}^{2} h} \frac{\partial}{\partial \tau}\left(\frac{1}{h} \frac{\partial Y_{n}}{\partial \tau}\right)\left(A_{n} \mathrm{e}^{\mu_{n} \breve{z}}+B_{n} \mathrm{e}^{-\mu_{n} \breve{z}}\right), \\
\breve{S}^{(0)}= & C_{0}-B_{0} \tau \\
& -\sum_{n=1}^{\infty} \frac{1}{\mu_{n} h} \frac{\partial}{\partial \tau}\left(\frac{1}{\bar{B}^{2} h} \frac{\partial}{\partial \tau} \frac{1}{h} \frac{\partial Y_{n}}{\partial \tau}-Y_{n}\right)\left(A_{n} \mathrm{e}^{\mu_{n} \breve{z}}-B_{n} \mathrm{e}^{-\mu_{n} \breve{z}}\right),
\end{aligned}
$$

where $C_{0}$ is another arbitrary constant.

To ensure that $\breve{S}^{(0)}$ is odd and periodic in $\tau$, we must take $B_{0}=C_{0}=0$. Applying the boundary condition (6.4) as $z \rightarrow \infty$, we must have $\breve{\Sigma}^{(0)} \rightarrow 0$ as $\breve{z} \rightarrow \infty$. This implies $A_{n}=0$ for all $n$.

\subsection{Matching the inner and outer solutions at $z=O(1)$}

Matching $\hat{N} \sim \mathscr{F} \breve{N}$ at $z=O(1)$, we must have $\hat{N}^{(0)} \rightarrow 0$ as $\hat{z} \rightarrow \infty$. Hence from (6.13) we need $n_{+}(\tau)=0$. Matching $\hat{\Sigma} \sim \breve{\Sigma}$ at $z=O(1)$, we must have $\hat{\Sigma}^{(0)}(\hat{z}, \tau) \rightarrow \breve{\Sigma}^{(0)}(0, \tau)$ as $\hat{z} \rightarrow \infty$. Hence from (6.14) and (6.26) we need $s_{\tau}(\tau)=0$, and also

$$
n_{-}(\tau)=v \sum_{n=1}^{\infty} B_{n} Y_{n}(\tau)
$$

Matching $\mathscr{F}^{-1 / 2} \hat{S} \sim \mathscr{F}^{1 / 2} \breve{S}$ at $z=O(1)$, we must have $\hat{S}^{(0)} \rightarrow 0$ as $\hat{z} \rightarrow \infty$. Hence from (6.15) we need $s(\tau)=0$.

Therefore the expressions for the variables in the inner boundary layer become

$$
\begin{aligned}
\hat{N}^{(0)} & =v \sum_{n=1}^{\infty} B_{n} Y_{n}(\tau) \mathrm{e}^{-|\bar{B}(\tau)| \hat{z}}, \\
\hat{\Sigma}^{(0)} & =\sum_{n=1}^{\infty} B_{n} Y_{n}(\tau), \\
\hat{S}^{(0)} & =0,
\end{aligned}
$$

while in the outer boundary layer, we have

$$
\begin{aligned}
\breve{N}^{(0)} & =\frac{1}{\bar{B}^{2}} \sum_{n=1}^{\infty} \frac{B_{n}}{h} \frac{\partial}{\partial \tau}\left(\frac{1}{h} \frac{\partial Y_{n}}{\partial \tau}\right) \mathrm{e}^{-\mu_{n} \breve{z}} \\
\breve{\Sigma}^{(0)} & =\sum_{n=1}^{\infty} B_{n} Y_{n}(\tau) \mathrm{e}^{-\mu_{n} \breve{z}} \\
\breve{S}^{(0)} & =\sum_{n=1}^{\infty} \frac{B_{n}}{\mu_{n} h} \frac{\partial}{\partial \tau}\left[\frac{1}{\bar{B}^{2} h} \frac{\partial}{\partial \tau}\left(\frac{1}{h} \frac{\partial Y_{n}}{\partial \tau}\right)-Y_{n}(\tau)\right] \mathrm{e}^{-\mu_{n} \breve{z}} .
\end{aligned}
$$




\subsection{The $O(\mathscr{F})$ corrections to $\hat{S}$ in the inner layer}

Since $\hat{S}^{(0)}=0$, it turns out that the leading-order displacement $\eta$ in the inner layer requires knowledge of the $O(\mathscr{F})$ correction $\hat{S}^{(1)}$. The solution for this function is most simply found as follows.

First we take the $O\left(\mathscr{F}^{1 / 2}\right)$ components of (A.45) with the inner-layer scalings (6.6)-(6.9) to obtain

$$
\hat{N}_{\tau}^{(0)}+\hat{S}_{\hat{z}}^{(1)}+2(1+v) \hat{S}_{\hat{z}}^{(0)}=0
$$

The two leading-order functions $\hat{N}^{(0)}$ and $\hat{S}^{(0)}$ are known from (6.30) and (6.32). We can then integrate with respect to $\hat{z}$, to obtain

$$
\hat{S}^{(1)}=v \sum_{n=1}^{\infty} \frac{B_{n}}{h} \frac{\partial}{\partial \tau}\left(\frac{Y_{n}(\tau)}{|\bar{B}(\tau)|} \mathrm{e}^{-|\bar{B}(\tau)| \hat{z}}\right)+C(\tau),
$$

for some function $C(\tau)$. We find $C(\tau)$ by matching with the outer solution as $\hat{z} \rightarrow \infty$. Examining the scalings, we see we must have $\hat{S}^{(1)} \sim \breve{S}^{(0)}$, and hence

$$
\lim _{\hat{z} \rightarrow \infty} \hat{S}^{(1)}=\lim _{\breve{z} \rightarrow 0} \breve{S}^{(0)}
$$

From (6.35), this sets

$$
C(\tau)=\sum_{n=1}^{\infty} \frac{B_{n}}{\mu_{n} h} \frac{\partial}{\partial \tau}\left[\frac{1}{\bar{B}^{2} h} \frac{\partial}{\partial \tau}\left(\frac{1}{h} \frac{\partial Y_{n}}{\partial \tau}\right)-Y_{n}(\tau)\right]
$$

and hence

$$
\hat{S}^{(1)}=\sum_{n=1}^{\infty} \frac{B_{n}}{h} \frac{\partial}{\partial \tau}\left\{\frac{v Y_{n}}{|\bar{B}|} \mathrm{e}^{-|\bar{B}| \hat{z}}+\frac{1}{\mu_{n}}\left[\frac{1}{|\bar{B}|^{2} h} \frac{\partial}{\partial \tau}\left(\frac{1}{h} \frac{\partial Y_{n}}{\partial \tau}\right)-Y_{n}\right]\right\}
$$

\subsection{Displacement recovery}

The displacements $\{\xi, \eta, \zeta\}$ can be recovered from the stress variables $\{\tilde{N}, \tilde{\Sigma}, \tilde{S}\}$ using the expressions (A.37)-(A.39). We find that in the inner boundary layer we have, to leading order in $\mathscr{F}$,

$$
\begin{aligned}
& \xi=\frac{\mathscr{F}^{1 / 2} h v}{12\left(1-v^{2}\right) \bar{B}(\tau)} \sum_{n=1}^{\infty} B_{n} Y_{n}(\tau)\left(1-\mathrm{e}^{-|\bar{B}| \hat{z}}\right), \\
& \eta=\frac{\mathscr{F}^{3 / 2}}{12\left(1-v^{2}\right)} \sum_{n=1}^{\infty} B_{n} \frac{\partial}{\partial \tau}\left\{Y_{n}(\tau)\left[\frac{v(2+v)}{|\bar{B}|^{2}}\left(1-\mathrm{e}^{-|\bar{B}| \hat{z}}\right)+\frac{v^{2} \hat{z}}{|\bar{B}|}-\frac{\hat{z}^{2}}{2}\right]\right. \\
& \left.+\frac{2(1+v)}{\mu_{n}}\left[\frac{1}{|\bar{B}|^{2} h} \frac{\partial}{\partial \tau}\left(\frac{1}{h} Y_{n}^{\prime}\right)-Y_{n}\right] \hat{z}\right\}, \\
& \zeta=\frac{\mathscr{F}}{12\left(1-v^{2}\right)} \sum_{n=1}^{\infty} B_{n} Y_{n}(\tau)\left[-\frac{v^{2}}{|\bar{B}|}\left(1-\mathrm{e}^{-|\bar{B}| \hat{z}}\right)+\hat{z}\right] \cdot a
\end{aligned}
$$

The scalings for $\tilde{N}, \tilde{\Sigma}$ and $\tilde{S}$ in the outer boundary layer, mean that the contributions to $\xi, \eta$ and $\zeta$ from there are asymptotically larger than those from the inner boundary layer, by a factor of at least $\mathscr{F}^{-1}$. Therefore in the outer boundary layer, there is no contribution to $\xi, \eta$ and $\zeta$ from the inner layer 
at leader order. From the integrals (A.37)-(A.39) over just the outer-layer stresses we obtain

$$
\begin{aligned}
& \xi=\frac{\mathscr{F}^{-1 / 2}}{12\left(1-v^{2}\right) \bar{B}(\tau)} \sum_{n=1}^{\infty} \frac{B_{n}}{\mu_{n}}\left(\frac{Y_{n}^{\prime}}{h}\right)^{\prime}\left[\frac{1}{\mu_{n}}\left(1-\mathrm{e}^{-\mu_{n} \breve{z}}\right)-\breve{z}\right], \\
& \eta=\frac{\mathscr{F}^{-1 / 2}}{12\left(1-v^{2}\right)} \sum_{n=1}^{\infty} \frac{B_{n}}{\mu_{n}} Y_{n}^{\prime}(\tau)\left[\frac{1}{\mu_{n}}\left(1-\mathrm{e}^{-\mu_{n} \breve{z}}\right)-\breve{z}\right], \\
& \zeta=\frac{1}{12\left(1-v^{2}\right)} \sum_{n=1}^{\infty} \frac{B_{n}}{\mu_{n}} Y_{n}(\tau)\left(1-\mathrm{e}^{-\mu_{n} \breve{z}}\right) .
\end{aligned}
$$

As $\breve{z} \rightarrow \infty$, the asymptotic forms of (6.44)-(6.46) are

$$
\begin{aligned}
& \xi \sim \frac{1}{12\left(1-v^{2}\right) \bar{B}} \sum_{n=1}^{\infty} \frac{B_{n}}{\mu_{n}}\left(\frac{Y_{n}^{\prime}}{h}\right)^{\prime}\left[\frac{\mathscr{F}^{-1 / 2}}{\mu_{n}}-z\right], \\
& \eta \sim \frac{1}{12\left(1-v^{2}\right)} \sum_{n=1}^{\infty} \frac{B_{n}}{\mu_{n}} Y_{n}^{\prime}(\tau)\left[\frac{\mathscr{F}^{-1 / 2}}{\mu_{n}}-z\right], \\
& \zeta \sim \frac{1}{12\left(1-v^{2}\right)} \sum_{n=1}^{\infty} \frac{B_{n}}{\mu_{n}} Y_{n}(\tau),
\end{aligned}
$$

where we have returned to the original axial variable $z=\mathscr{F}^{-1 / 2} \breve{z}$ to aid comparison with the earlier work on matching to the interior solution. We observe that we obtain the expected behaviours (4.11)(4.13): $\zeta$ tends to a function of $\tau$, while $\xi$ and $\eta$ tend to a function of $\tau$ plus $z$ times a function of $\tau$. Moreover, each mode is consistent with the $z \rightarrow \infty$ asymptotic forms (5.2)-(5.4) of the eigenvalue problem specified in $\S 5.2$, if we take

$$
f_{n}(\tau)=Y_{n}(\tau), \quad z_{n}^{*}=\frac{\mathscr{F}^{-1 / 2}}{\mu_{n}}
$$

Using the expressions (6.25) for the limit of a circular cross-section, we obtain

$$
f_{n}(\tau) \sim \cos (2 n \tau), \quad z_{n}^{*} \sim \frac{\mathscr{F}^{-1 / 2}}{2 n \sqrt{4 n^{2}+1}} \quad \text { as } \quad \sigma_{0} \rightarrow \infty .
$$

These expressions agree with the $\mathscr{F} \ll 1$ limit of the solution (A.28) for a circular cross-section found in Appendix C.

\subsection{Boundary-layer structure}

We observe that the inner layer is entirely passive at leading order in the limit $\mathscr{F} \rightarrow 0$. It does not impose any azimuthal structure on the solution or contribute to the leading-order displacements as $z \rightarrow \infty$. The inner layer simply allows the decay of the azimuthal hoop stress $\tilde{N}$ from its $O\left(\mathscr{F}^{1 / 2}\right)$ value at $z=0$ to the $O\left(\mathscr{F}^{3 / 2}\right)$ value needed in the outer layer, while leaving $\tilde{\Sigma}$ and $\tilde{S}$ approximately constant in $z$. The dominant physical effects in the inner layer are the normal forces that arise from the azimuthal hoop stress and axial tension interacting with the tube curvature.

The outer layer allows for the decay of the axial stress $\tilde{\Sigma}$ and shear stress $\tilde{S}$, along with the growth of the axial displacement $\zeta$ for matching with the interior solution as $z \rightarrow \infty$. It is the outer layer that 
determines the azimuthal modes $Y_{n}(\tau)$. The dominant physical effects in the outer layer are forces from the axial stress and shear stress, together with the curvature effects that were also present in the inner layer.

Some aspects of this structure can be seen in figure A.8 in Appendix C which deals with the case of a circular cross section. The graphs in the figure show the magnitudes of the $n=1$ modes of the displacements and stresses as functions of the axial coordinate.

\section{Boundary conditions for the interior solution}

\subsection{Matching to $\check{\eta}$ in the bulk interior}

Substituting (6.50) in to (5.5), we obtain the boundary conditions on the interior solution for $\check{\eta}(\tau, \check{z})$ as

$$
\check{\eta}=\sum_{n=1}^{\infty} G_{n} \frac{\mathscr{F}^{-1 / 2}}{\ell \mu_{n}} Y_{n}^{\prime}(\tau) \quad \text { and } \quad \frac{\partial \check{\eta}}{\partial \check{z}}=-\sum_{n=1}^{\infty} G_{n} Y_{n}^{\prime}(\tau) \quad \text { at } \quad \check{z}=0,
$$

We can eliminate the constants $G_{n}$ using the orthogonality of the $Y_{n}(\tau)$. We define

$$
W_{n}=\frac{1}{h} \frac{\partial}{\partial \tau}\left(\frac{1}{\bar{B}^{2} h} \frac{\partial}{\partial \tau} \frac{1}{h} \frac{\partial}{\partial \tau}-1\right) Y_{n}
$$

so that $h \mathscr{L} Y_{n}=\partial W_{m} / \partial \tau$. Then, using integration by parts, we have

$$
\begin{aligned}
\int_{0}^{2 \pi} Y_{n}^{\prime}(\tau) W_{m}(\tau) \mathrm{d} \tau & =-\int_{0}^{2 \pi} Y_{n} \frac{\partial W_{m}}{\partial \tau} \mathrm{d} \tau \\
& =-\int_{0}^{2 \pi} Y_{n}\left(\mathscr{L} Y_{m}\right) h \mathrm{~d} \tau \\
& =-\mu_{m} \int_{0}^{2 \pi} Y_{n} Y_{m} h \mathrm{~d} \tau=-\delta_{n m} \mu_{n} D_{n}
\end{aligned}
$$

for some set of normalisation constants $D_{n}$.

We now multiply each of (7.1) by $W_{m}(\tau)$ and integrate between 0 and $2 \pi$ to extract the individual modes. Eliminating $G_{m}$ between each pair of equations gives us

$$
\int_{0}^{2 \pi}\left(\check{\eta}+\frac{1}{\mu_{m} \mathscr{F} 1 / 2 \ell} \frac{\partial \check{\eta}}{\partial \check{z}}\right) W_{m}(\tau) \mathrm{d} \tau=0 \quad \text { at } \quad \check{z}=0 \quad \text { for each } m \text {. }
$$

The corresponding boundary condition for a boundary layer near $\check{z}=1$ would be

$$
\int_{0}^{2 \pi}\left(\check{\eta}-\frac{1}{\mu_{m} \mathscr{F} 1 / 2 \ell} \frac{\partial \check{\eta}}{\partial \check{z}}\right) W_{m}(\tau) \mathrm{d} \tau=0 \quad \text { at } \quad \check{z}=1 \quad \text { for each } m \text {. }
$$

\subsection{Boundary conditions for the tube law}

The boundary conditions (7.4) and (7.5) derived above would be applied to $\check{\eta}(\tau, \check{z})$ when the PDE (2.1) is solved for the deformations in the bulk interior of the tube. However, we recall from $\S 2$ that rather than solving the PDE for $\check{\eta}(\tau, \check{z})$, Whittaker et al. (2010b) found an approximate solution by assuming that the deformations are dominated by a fundamental azimuthal mode. Writing $\check{\eta} \approx b(\check{z}) \sin (2 \tau)$, the tube law (2.2) was obtained:

$$
k_{2} \tilde{F} \frac{\partial^{2} \alpha}{\partial \check{z}^{2}}-k_{0} \alpha=-\tilde{P}(\check{z})
$$


where $\alpha(\check{z}) \propto b(\check{z})$ is the dimensionless change in cross-sectional area of the tube, $\tilde{P}$ is the dimensionless transmural pressure, and the $k_{i}$ are numerically determined constants that depend on the ellipticity of the undeformed tube. Since the azimuthal dependence of the deformation is now fixed, we are unable to apply more than one of the conditions (7.4) and (7.5) at each end.

We now assume that the fundamental mode $\sin (2 \tau)$ in the interior is sufficiently close to $Y_{1}^{\prime}(\tau)$ for the boundary conditions (7.4) and (7.5) to be dominated by the $m=1$ equation. (The agreement is perfect in the limit $\sigma \rightarrow \infty$ and, as can be seen in figure $4 b$, the mode shape of $Y_{n}(\tau)$ is still fairly sinusoidal even for much smaller $\sigma$.) Under this assumption, the boundary conditions to be applied to $\alpha(\check{z})$ when $\mathscr{F} \ll 1$ are

$$
\check{z}^{*} \frac{\partial \alpha}{\partial \check{z}}+\alpha=0 \quad \text { at } \quad \check{z}=0, \quad \check{z}^{*} \frac{\partial \alpha}{\partial \check{z}}-\alpha=0 \quad \text { at } \quad \check{z}=1 .
$$

where $\check{z}^{*}=\left(\mu_{1} \mathscr{F}^{1 / 2} \ell\right)^{-1}$.

\section{Comparison with numerical simulations}

We now construct solutions for $\alpha(\check{z})$ to the tube-law model (2.2) for the case of a tube subject to a uniform transmural pressure, both with with the original tube-end boundary conditions (2.3) and with the modified conditions (7.7) resulting from the boundary-layer analysis. These results are then compared with numerical simulations of the same tube in which the full Kirchhoff-Love shell equations are solved using a finite-element scheme.

\subsection{Numerical simulations}

Numerical simulations were performed using the Oomph-lib finite-element framework (Heil \& Hazel, 2007). In each simulation, the dimensionless Kirchhoff-Love shell equations were solved to find the deformation of an initially elliptical pre-stressed tube with given tube-end boundary conditions under a prescribed transmural pressure. Full details of the method can be found in in Whittaker et al. (2010b).

In all the simulations, we considered a tube with initial ellipticity $\sigma_{0}=0.6$ and Poisson ratio $v=$ 0.49 , subject to a dimensionless axial tension $\tilde{F}=1$, and uniform transmural pressure $\tilde{P}=-1$. We used a range of different values of the dimensionless wall thickness $\delta$ and tube length $\ell$, resulting in different tension parameters $\mathscr{F}$. For each set of parameters, we performed a simulation with each of the three different tube-end boundary conditions: sliding, pinned and clamped (as described in $\S 2$ ).

\subsection{Analytical solutions to the tube-law models}

With $\tilde{P}=-1$, the solution to the tube law (7.6) subject to the original boundary conditions to $\alpha=0$ at $\check{z}=0,1$ is

$$
\alpha(\check{z})=-\frac{1}{k_{0}}\left(1-\frac{\cosh \lambda\left(\check{z}-\frac{1}{2}\right)}{\cosh (\lambda / 2)}\right),
$$

where $\lambda=\left(k_{2} \tilde{F} / k_{0}\right)^{-1 / 2}$.

The solution of (7.6) subject to the new conditions (7.7) is given by

$$
\alpha(\check{z})=-\frac{1}{k_{0}}\left(1-\frac{\cosh \lambda\left(\check{z}-\frac{1}{2}\right)}{\cosh (\lambda / 2)-\check{z}^{*} \lambda \sinh (\lambda / 2)}\right) .
$$

Observe that as $\check{z}^{*} \rightarrow 0$, this solution returns to the original solution (8.1). 
The relative area change $\alpha$ is proportional to $\int_{0}^{2 \pi} \xi \mathrm{d} \tau$. So in the interior, $\alpha$ is proportional to amplitude of the fundamental azimuthal mode of $\check{\eta}$ to good approximation. In the boundary layers, $\alpha$ will be proportional to the $z$-dependence in the amplitude of the fundamental mode for $\xi$ in (6.44) to good approximation. In the inner boundary layer, the smaller $O\left(\mathscr{F}^{1 / 2}\right)$ scale for $\xi$ in $(6.41)$ means that the area changes here are negligible compared with those in the outer layer and interior.

We can therefore obtain an approximate expression $\alpha_{\mathrm{bl}}(\check{z})$ for $\alpha(\check{z})$ in the boundary layer using the axial dependence of the $n=1$ mode in (6.44). The constant is most easily determined by matching to (8.2) for $\check{z}^{*} \ll \check{z} \ll 1$. Near $\check{z}=0$ we have

$$
\alpha_{\mathrm{bl}}(\check{z})=\frac{1}{k_{0}}\left(\frac{-\lambda \sinh (\lambda / 2)}{\cosh (\lambda / 2)-\check{z}^{*} \lambda \sinh (\lambda / 2)}\right)\left(\check{z}-\check{z}^{*}\left[1-\mathrm{e}^{-\check{z} / \check{z} *}\right]\right) .
$$

Near $\check{z}=1$, the boundary-layer solution will be $\alpha_{\mathrm{bl}}(1-\check{z})$ by symmetry.

We can now construct a composite solution $\alpha_{\mathrm{c}}(\check{z})$ valid over the whole length of the tube. This is obtained by summing the two expressions for $\alpha$ and $\alpha_{\mathrm{bl}}$, and then subtracting off the common linear parts in the two matching regions (see, for example, Hinch, 1991, chapter 5). Thus

$$
\begin{aligned}
& \alpha_{\mathrm{c}}(\check{z})=\alpha(\check{z})+\alpha_{\mathrm{bl}}(\check{z})+\alpha_{\mathrm{bl}}(1-\check{z}) \\
&+\left(\frac{\lambda k_{0}^{-1} \sinh (\lambda / 2)}{\cosh (\lambda / 2)-\check{z}^{*} \lambda \sinh (\lambda / 2)}\right)\left(\check{z}-\check{z}^{*}+(1-\check{z})-\check{z}^{*}\right), \\
&=-\frac{1}{k_{0}}\left(1-\frac{\cosh \lambda\left(\check{z}-\frac{1}{2}\right)-\check{z}^{*} \lambda \sinh (\lambda / 2)\left(\mathrm{e}^{-\check{z} / \check{z}^{*}}+\mathrm{e}^{-(1-\check{z}) / \check{z}^{*}}\right)}{\cosh (\lambda / 2)-\check{z}^{*} \lambda \sinh (\lambda / 2)}\right) .
\end{aligned}
$$

However, we note that this solution does not precisely satisfy the $\alpha=0$ boundary conditions at $\check{z}=0,1$ because of the tails from the boundary layers at the opposite ends. When $\check{z}^{*} \ll 1$ the errors will be exponentially small, but they could be significant if $1 / z^{*} \geqslant O(1)$. (Though in the latter case, it is not really appropriate to use the boundary layer expansion.)

To account for the error in the boundary conditions (and potentially expand the range of validity of the solution) we can construct an $a d$-hoc correction by altering the coefficients of the four exponential functions $\left(\mathrm{e}^{ \pm \lambda \check{z}}, \mathrm{e}^{ \pm \check{z} / \check{z}^{*}}\right)$ in $\alpha_{\mathrm{c}}$ in order that it satisfies $\alpha_{\mathrm{c}}=0$ and $\alpha_{\mathrm{c}}^{\prime}=0$ at $\check{z}=0,1$. The resulting function is

$$
\alpha_{\mathrm{c}}(\check{z})=-\frac{1}{k_{0}}\left(1-\frac{\left.\cosh \lambda\left(\check{z}-\frac{1}{2}\right)-\check{z}^{*} \lambda \sinh (\lambda / 2) \frac{\cosh \left((\check{z}-1 / 2) / \check{z}^{*}\right)}{\sinh \left(1 / 2 \check{z}^{*}\right)}\right)}{\cosh (\lambda / 2)-\check{z}^{*} \lambda \sinh (\lambda / 2) \operatorname{coth}\left(1 / 2 \check{z}^{*}\right)}\right) .
$$

When $\check{z}^{*} \ll 1$ this reduces to the original expression (8.4), as would be expected.

For $\sigma_{0}=0.6$, the numerical parameters needed for the theoretical solutions are $k_{0}=11.075, k_{2}=$ 1.7044 (from Whittaker et al., 2010b) and $\mu_{1}=5.5294$ (from $\S 6.2$ above). Combined with $v=0.49$ and $\tilde{F}=1.0$, we then have

$$
\begin{aligned}
& \mathscr{F}=\frac{\delta^{2} \ell^{2} \tilde{F}}{12\left(1-v^{2}\right)} \\
& \lambda=\left(\frac{k_{0}}{k_{2} \tilde{F}}\right)^{1 / 2} \\
& \check{z}^{*}=\frac{\mathscr{F}^{-1 / 2}}{\mu_{1} \ell} \\
& =0.1097 \delta^{2} \ell^{2} \text {, } \\
& =2.549 \text {, } \\
& =0.5461 \delta^{-1} \ell^{-2} \text {. }
\end{aligned}
$$

The fact that $\lambda$ is independent of $\delta$ and $\ell$ means that the original tube-law solution (8.1) will be identical in all cases. 
(a)

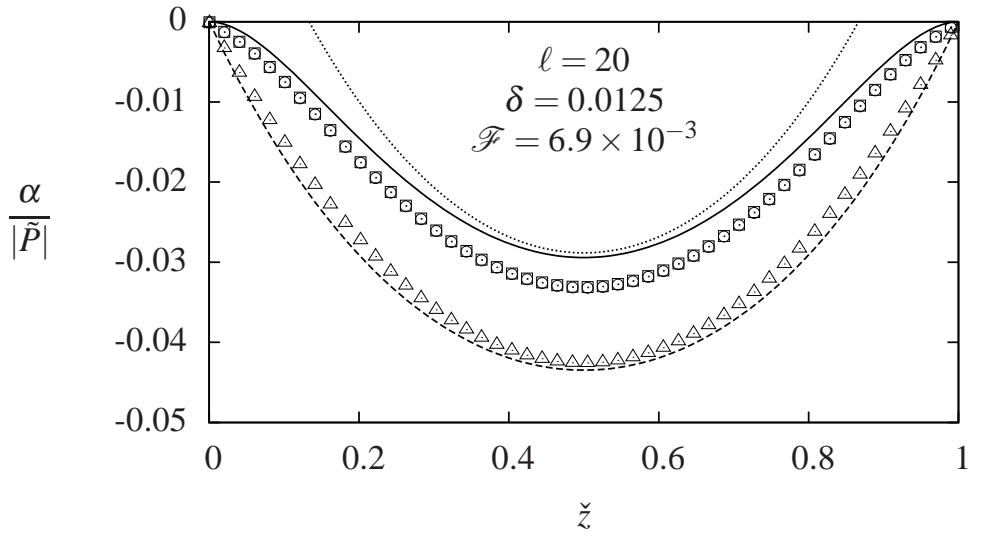

(b)

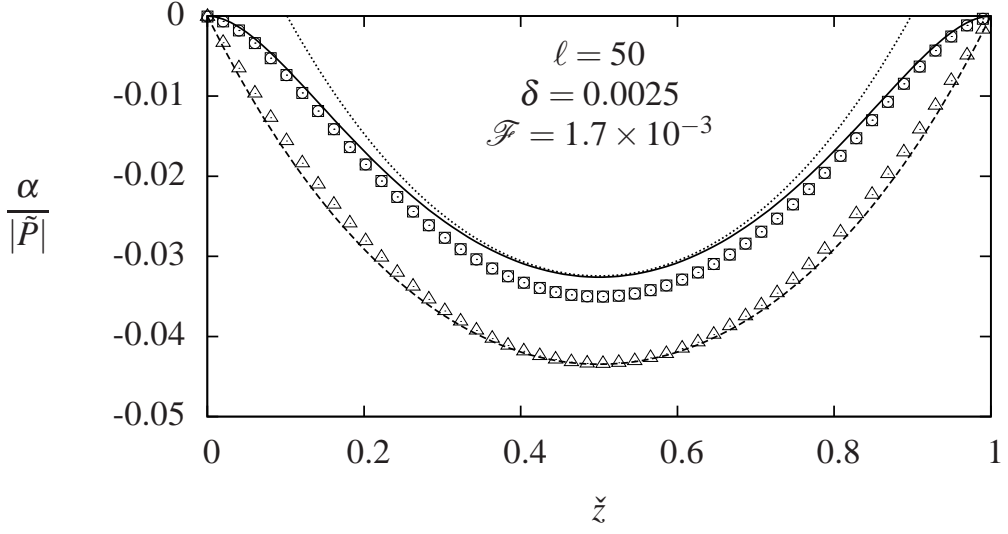

(c)

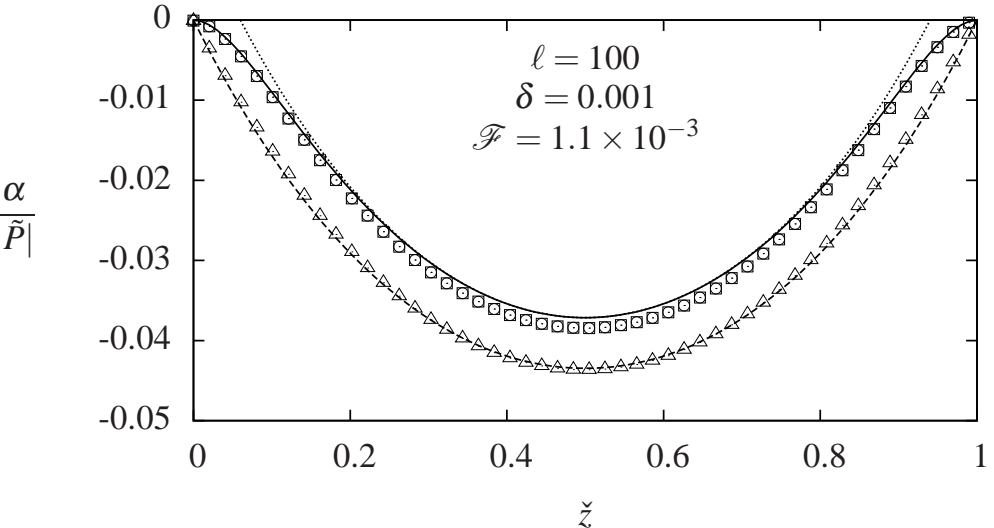

FIG. 6. Comparison of 3D shell-theory with results from the shear-layer model. Points show numerical shell-theory results for a buckled tube with clamped (squares), pinned (circles) and sliding (triangles) boundary conditions. Lines show the original tube-law solution (8.1) (dashed), the composite solution (8.5) from the shear-layer model (continuous), and the interior solution (8.2) from the shear-layer model (dotted). All calculations have $\tilde{F}=1.0$ and $v=0.49$. 


\subsection{Comparison}

Graphs comparing some typical numerical simulations along with the original tube-law solution (8.1) and the composite solution (8.5) from the shear-layer model are shown in figure 6. As can be seen, the original tube law solution (8.1) is a good fit to the numerical results with the sliding boundary conditions, but not to those with the other two conditions. The numerical solutions with clamped and pinned boundary conditions are almost indistinguishable. The new composite solutions (8.5) using the boundary-layer model fit these solutions reasonably well, and appear to be capturing the essential physics.

The least good fit is seen in figure 6(a), which has the shortest $\ell$ and the largest $\mathscr{F}$, both of which would be expected to decrease the accuracy of the asymptotic solutions. It also has the largest off-set $\check{z}^{*}$, to the point where the boundary layers induced at each end of the tube significantly overlap one another. Given these factors, and the various approximations made in the tube-law and boundary-layer models, the agreement with the numerical simulations in this case is still reasonable.

\section{Summary and Conclusions}

In this paper, we have formulated a boundary-layer description for the deformation of an initially elliptical elastic-walled tube near an end that is subject to pinned boundary conditions. The boundary-layer includes in-plane shear effects that are negligible in the interior bulk solution. The boundary-layer system (4.5)-(4.7) for for the displacements $\xi, \eta, \zeta$ must be solved subject to the boundary conditions (4.8) at the pinned boundary, along with the far-field matching conditions (4.11)-(4.13) and (4.14).

The original PDE system (2.1) in the interior is only second-order in $z$, which allowed only one condition to be satisfied at the boundary. The boundary-layer system (4.5)-(4.7) is 6th-order in the axial coordinate $z$, which allows the full set of three pinned conditions (4.8) to be satisfied. The additional terms present in the boundary-layer equations correspond to forces arising from in-plane shearing.

We have shown how the boundary-layer system can be formulated as an eigenvalue problem independently of the interior solution. The effective boundary conditions on the interior solution then take the form of a set of Robin conditions (5.6) on the eigenmode components of $\check{\eta}$. It is seen that the boundary layer only has a significant affect on the boundary conditions that the interior solution sees when the dimensionless parameter $\mathscr{F}$ (as defined in (4.4)) is much less than one. Physically, this corresponds to axial-tension-curvature effects being weak compared with in-plane stretching on the scale of the tube diameter.

A matched asymptotic expansion of the boundary-layer solution for $\mathscr{F} \ll 1$ reveals a double-layer structure: an inner layer with thickness $O\left(\mathscr{F}^{1 / 2}\right)$ and an outer layer with thickness $O\left(\mathscr{F}^{-1 / 2}\right)$. The leading-order solutions are expressed in terms of the eigenfunctions of a one-dimensional linear operator (6.24), which are determined numerically. The boundary conditions on the interior solution, can then be expressed as a set of integral constraints (7.4).

The tube law (2.2) used previously in place of the full PDE system in the interior assumes that the deformations are dominated by a fundamental azimuthal mode. Except when the tube is circular, this mode is not identical to the fundamental eigenmode in the boundary layer. Nevertheless it is similar, and we obtain the approximate boundary conditions (7.7) on the interior tube-law solution by just matching it to the fundamental boundary-layer eigenmode. Composite solutions including the boundary layer can also be formed. Analytical results using this approximate matching are shown to compare well with full numerical simulations. As shown in figure 6, the new composite solutions fit the numerical data much better that the previous tube-law solutions when $\mathscr{F} \ll 1$.

The agreement between the composite solution and the numerical results improves noticeably as 
the tube length $\ell$ is increased. The poorer agreement at smaller $\ell$ is probably due to a combination of not being as well inside the $\ell \ll 1$ asymptotic regime and also the interactions between the two boundary layers from the two ends of the tube. (It can be seen clearly in figure $6 a$ that the boundarylayer corrections have not quite decayed by $\check{z}=0.5$ in the middle of the tube.)

Two other features of the solution are worth drawing attention to. First, the nature of the boundary layer we have described is somewhat unusual. It arises not because of a direct local effect at each point on the tube wall, but because of non-local geometrical constraint around its whole circumference. It is the effect of the shear constraint in the bulk interior being integrated around the circumference, that creates the need for the boundary layer.

Secondly, the boundary-layer model derived here only accounts for the shear forces, to allow pinned boundary conditions to be satisfied. It does not include axial bending forces, and hence it is still not possible to satisfy the full clamped boundary conditions at the tube ends. However, examining the displacements (6.44)-(6.46) in the outer boundary layer, we see that the leading-order terms have the following behaviour as we head towards the inner layer:

$$
\xi \sim \breve{z}^{2}, \quad \eta \sim \breve{z}^{2}, \quad \zeta \sim \breve{z} \quad \text { as } \quad \breve{z} \rightarrow 0 .
$$

Therefore the inner limit of the outer boundary-layer solution already satisfies the 'clamped' conditions $(\xi=\eta=\zeta=\partial \xi / \partial z=0)$ at leading order. Therefore any bending boundary layer is likely to be weaker than might be assumed on simple scaling grounds. This may well help explain why the numerical simulations show negligible difference between the solutions with pinned and clamped boundary conditions.

Finally, we note that this work was carried out for a tube with an initially elliptical cross-section, to aid comparison with previous studies. The shape of the initial cross-section enters via the base-state azimuthal curvature $\bar{B}$ in the operator $\mathscr{L}$ as defined in (6.24), and hence has an effect of the azimuthal deformation modes $Y_{n}(\tau)$ in the boundary layers. Physically the initial cross-section shape matters because of the forces that arise from the product of the base-state curvature $\bar{B}$ and the azimuthal hoop stress perturbation $\tilde{N}$.

The limiting case of a circular cross-section is approached smoothly, and there are no singular changes to the boundary layers in this limit. (This is in contrast to the case of oscillations in elastic walled tubes, where the deformation-area relationship for almost circular initial cross-sections is significantly different, and would have a profound effect on the dynamics.) It would be relatively straightforward to repeat the calculations here to derive the boundary-layer modes $Y_{n}(\tau)$ for any other initial cross-sectional shape.

\section{Acknowledgements}

The author gratefully acknowledges Prof M Heil of the University of Manchester for performing the numerical simulations shown in figure 6 using oomph-1ib.

\section{REFERENCES}

Bertram, C. D. \& Pedley, T. J. 1982 A mathematical model of unsteady collapsible tube behaviour. J. Biomech. 15 (1), 39-50.

Flaherty, J. E., Keller, J. \& Rubinow, S. I. 1972 Post-buckling behaviour of elastic tubes and rings with opposite sides in contact. SIAM J. Appl. Math. 23, 446-455.

FLÜGGE, W. 1972 Tensor Analysis and Continuum Mechanics. Springer-Verlag. 
Grotberg, J. B. \& Jensen, O. E. 2004 Biofluid mechanics in flexible tubes. Annu. Rev. Fluid Mech. 36, 121147.

HeIL, M. \& HAZEL, A. 2007 Oomph-lib — an object-oriented multi-physics finite-element library. Open-source software; available online from http://www.oomph-lib.org/.

HeIL, M. \& HAZEL, A. L. 2011 Fluid-structure interaction in internal physiological flows. Annu. Rev. Fluid Mech. 43, 141-162.

Heil, M. \& Jensen, O. E. 2003 Flows in deformable tubes and channels: Theoretical models and biological applications. In Flow Past Highly Compliant Boundaries and in Collapsible Tubes (ed. P. W. Carpenter \& T. J. Pedley), chap. 2, pp. 15-49. Kluwer Academic, Dordrecht.

Hinch, E. J. 1991 Perturbation Methods. Cambridge University Press.

McClurken, M. E., Kececioglu, I., Kamm, R. D. \& Shapiro, A. H. 1981 Steady, supercritical flow in collapsible tubes. Part 2. Theoretical studies. J. Fluid Mech. 109, 391-415.

REYN, J. W. 1987 Multiple solutions and flow limitation for steady flow through a collapsible tube held open at the ends. J. Fluid Mech. 174, 467-493.

SHAPIRO, A. H. 1977 Steady flow in collapsible tubes. ASME J. Biomech. Engr 99, 126-147.

StewArt, P. S., WATERS, S. L. \& JenSEn, O. E. 2009 Local and global instabilities of flow in a flexible-walled channel. Eur. J. Mech. B/Fluids 28 (4), 541-557.

Whittaker, R. J., Waters, S. L., Jensen, O. E., Boyle, J. \& Heil, M. $2010 a$ The energetics of flow through a rapidly oscillating tube. Part 1. General theory. J. Fluid Mech. 648, 83-121.

Whittaker, R. J., Heil, M., Jensen, O. E. \& Waters, S. L. $2010 b$ A rational derivation of a tube law from shell theory. Q. J. Mech. Appl. Math. 63 (4), 465-496.

\section{A. Curvature, strain and covariant derivatives}

In this appendix, we evaluate the components of the metric tensor $a_{\alpha \beta}$, the curvature tensor $b_{\alpha \beta}$, the inplane strain $\gamma_{\alpha \beta}$, and the bending strain $\kappa_{\alpha \beta}$ in terms of the deformation functions $\xi, \eta$ and $\zeta$ introduced in (3.16). We also evaluate the covariant derivatives $\nabla_{\alpha}$ in terms of the partial derivatives $\partial / \partial \tau$ and $\partial / \partial z$.

We first define the set of orthogonal unit vectors $\{\hat{\boldsymbol{t}}, \hat{\boldsymbol{z}}, \hat{\boldsymbol{n}}\}$ in the azimuthal, axial, and normal directions with respect to the undeformed surface $\overline{\boldsymbol{r}}(\tau, z)$ of the tube in (3.2):

$$
\hat{\boldsymbol{t}}=\frac{c}{h(\tau)}\left(\begin{array}{c}
-\cosh \sigma_{0} \sin \tau \\
\sinh \sigma_{0} \cos \tau \\
0
\end{array}\right), \quad \hat{z}=\left(\begin{array}{l}
0 \\
0 \\
1
\end{array}\right), \quad \hat{\boldsymbol{n}}=\frac{c}{h(\tau)}\left(\begin{array}{c}
\cosh \sigma_{0} \cos \tau \\
\sinh \sigma_{0} \sin \tau \\
0
\end{array}\right)
$$

where $c$ is the normalisation constant defined in (3.3) and $h(\tau)$ is the scale factor defined in (3.5). We also define the dimensionless azimuthal curvature in the undeformed state by

$$
\bar{B} \equiv a \hat{\boldsymbol{n}} \cdot \frac{\partial \hat{\boldsymbol{t}}}{\partial x^{1}}=\frac{1}{h} \hat{\boldsymbol{n}} \cdot \frac{\partial \hat{\boldsymbol{t}}}{\partial \tau}=-\frac{c^{2} \sinh 2 \sigma_{0}}{2 h^{3}}
$$

We now compute the basis vectors $\boldsymbol{a}_{i}$ in the deformed configuration from the displacement $\boldsymbol{r}$ in 
(3.16):

$$
\begin{aligned}
& \boldsymbol{a}_{1} \equiv \frac{\partial \boldsymbol{r}}{\partial x^{1}}=\hat{\boldsymbol{t}}+\frac{\varepsilon}{\ell h}\left[\left(-\xi \bar{B}+\frac{\partial}{\partial \tau}\left(\frac{\eta}{h}\right)\right) \hat{\boldsymbol{t}}+\left(\eta \bar{B}+\frac{\partial}{\partial \tau}\left(\frac{\xi}{h}\right)\right) \hat{\boldsymbol{n}}\right]+\frac{\varepsilon}{\ell h}\left[\frac{\partial \zeta}{\partial \tau} \hat{z}\right]+O\left(\varepsilon^{2}\right), \\
& \boldsymbol{a}_{2} \equiv \frac{\partial \boldsymbol{r}}{\partial x^{2}}=\hat{\boldsymbol{z}}+\frac{\varepsilon}{\ell}\left[\frac{1}{h} \frac{\partial \xi}{\partial z} \hat{\boldsymbol{n}}+\frac{1}{h} \frac{\partial \eta}{\partial z} \hat{\boldsymbol{t}}+\frac{\partial \zeta}{\partial z} \hat{z}\right]+O\left(\varepsilon^{2}\right) \\
& \boldsymbol{a}_{3} \equiv \frac{\boldsymbol{a}_{1} \times \boldsymbol{a}_{2}}{\left|\boldsymbol{a}_{1} \times \boldsymbol{a}_{2}\right|}=\hat{\boldsymbol{n}}-\frac{\varepsilon}{\ell h}\left[\left(\eta \bar{B}+\frac{\partial}{\partial \tau}\left(\frac{\xi}{h}\right)\right) \hat{\boldsymbol{t}}+\frac{\partial \xi}{\partial z} \hat{\boldsymbol{z}}\right]+O\left(\varepsilon^{2}\right)
\end{aligned}
$$

The components of the metric tensor $a_{\alpha \beta}$ are then found to be

$$
\begin{aligned}
a_{11} & \equiv \boldsymbol{a}_{1} \cdot \boldsymbol{a}_{1}=1+\frac{2 \varepsilon}{\ell h}\left[-\xi \bar{B}+\frac{\partial}{\partial \tau}\left(\frac{\eta}{h}\right)\right]+\ldots, \\
a_{12}=a_{21} & \equiv \boldsymbol{a}_{1} \cdot \boldsymbol{a}_{2}=\frac{\varepsilon}{\ell h}\left[\frac{\partial \eta}{\partial z}+\frac{\partial \zeta}{\partial \tau}\right]+\ldots, \\
a_{22} & \equiv \boldsymbol{a}_{2} \cdot \boldsymbol{a}_{2}=1+\frac{2 \varepsilon}{\ell}\left[\frac{\partial \zeta}{\partial z}\right]+\ldots,
\end{aligned}
$$

and those of the curvature tensor $b_{\alpha \beta}$ are

$$
\begin{aligned}
b_{11} & \equiv \boldsymbol{a}_{3} \cdot \frac{\partial \boldsymbol{a}_{1}}{\partial x^{1}}=\frac{\bar{B}}{a}+O\left(\frac{\varepsilon}{a \ell}\right), \\
b_{12}=b_{21} & \equiv \boldsymbol{a}_{3} \cdot \frac{\partial \boldsymbol{a}_{2}}{\partial x^{1}}=O\left(\frac{\varepsilon}{a \ell}\right) \\
b_{22} & \equiv \boldsymbol{a}_{3} \cdot \frac{\partial \boldsymbol{a}_{2}}{\partial x^{2}}=\frac{\varepsilon}{a \ell h} \frac{\partial^{2} \xi}{\partial z^{2}}+\ldots
\end{aligned}
$$

By setting $\varepsilon=0$ in (A.6)-(A.11), we obtain expressions for the tensors $\bar{a}_{\alpha \beta}$ and $\bar{b}_{\alpha \beta}$ that describe the undeformed state.

We now use the definitions (3.12) of $\gamma_{\alpha \beta}$ and $\kappa_{\alpha \beta}$ to obtain the leading-order expressions

$$
\begin{aligned}
\gamma_{11} & =\frac{\varepsilon}{\ell h}\left(-\xi \bar{B}+\frac{\partial}{\partial \tau}\left(\frac{\eta}{h}\right)\right), \\
\gamma_{12}=\gamma_{21} & =\frac{1}{2} \frac{\varepsilon}{\ell h}\left(\frac{\partial \eta}{\partial z}+\frac{\partial \zeta}{\partial \tau}\right), \\
\gamma_{22} & =\frac{\varepsilon}{\ell} \frac{\partial \zeta}{\partial z}
\end{aligned}
$$

and

$$
\kappa_{11}, \kappa_{12}, \kappa_{21}, \kappa_{22}=O\left(\frac{\varepsilon}{a \ell}\right) .
$$

Finally, we need to evaluate the covariant derivative of a tensor. The expression for the inner covariant derivative of a second-rank tensor is given in (3.25). The Christoffel symbol is defined by

$$
\Gamma_{\mu v}^{\beta} \equiv a^{\alpha \beta} \boldsymbol{a}_{\alpha} \cdot \frac{\partial \boldsymbol{a}_{\mu}}{\partial x^{v}}
$$


which is symmetric in $\mu$ and $v$. In the undeformed state, it is easy to see that all the components of $\Gamma_{\mu v}^{\beta}$ are zero. Hence $\Gamma_{\mu v}^{\beta}=O(\varepsilon)$. Since we neglect $O\left(\varepsilon^{2}\right)$ quantities, the only components of $\Gamma_{\mu v}^{\beta}$ that we need are those that will be multiplied by an $O\left(\varepsilon^{0}\right)$ quantity. The only such quantity present in a covariant derivative is the pre-stress in $N^{22}$. Examining (3.25), we see that the components needed are $\Gamma_{21}^{1}, \Gamma_{22}^{1}$ and $\Gamma_{22}^{2}$. These are evaluated, to $O(\varepsilon)$, as follows:

$$
\begin{gathered}
\Gamma_{21}^{1}=\Gamma_{12}^{1}=a^{\alpha 1} \boldsymbol{a}_{\alpha} \cdot \frac{\partial \boldsymbol{a}_{1}}{\partial x^{2}}=\boldsymbol{a}_{1} \cdot \frac{\partial \boldsymbol{a}_{1}}{\partial x^{2}}+O\left(\varepsilon^{2}\right) \\
=\frac{\partial}{\partial x^{2}}\left(\frac{1}{2} \boldsymbol{a}_{1} \cdot \boldsymbol{a}_{1}\right)+O\left(\varepsilon^{2}\right)=\frac{1}{a} \frac{\partial \gamma_{11}}{\partial z}+O\left(\varepsilon^{2}\right) \\
\Gamma_{22}^{1}=a^{\alpha 1} \boldsymbol{a}_{\alpha} \cdot \frac{\partial \boldsymbol{a}_{2}}{\partial x^{2}}=\hat{\boldsymbol{t}} \cdot \frac{\partial \boldsymbol{a}_{2}}{\partial x^{2}}+O\left(\varepsilon^{2}\right)=\frac{\varepsilon}{a h} \frac{\partial^{2} \eta}{\partial z^{2}}+O\left(\varepsilon^{2}\right), \\
\Gamma_{22}^{2}=a^{\alpha 2} \boldsymbol{a}_{\alpha} \cdot \frac{\partial \boldsymbol{a}_{2}}{\partial x^{2}}=\hat{z} \cdot \frac{\partial \boldsymbol{a}_{2}}{\partial x^{2}}+O\left(\varepsilon^{2}\right)=\frac{\varepsilon}{a} \frac{\partial^{2} \zeta}{\partial z^{2}}+O\left(\varepsilon^{2}\right),
\end{gathered}
$$

where we have made use of the definition (3.12) of $\gamma_{11}$, and the expressions (A.3) and (A.4) for $\boldsymbol{a}_{1}$ and $a_{2}$.

\section{B. Verifying the number of boundary conditions}

In this appendix we argue that we do indeed have the correct number of boundary constraints for the number of degrees of freedom in the combined boundary-layer-interior system that is described in $\S 4.4$ and in the eigenvalue problem described in $\S 5.2$.

\section{B.1 Boundary-layer-interior system of $\S 4.4$}

Considering just one end of the tube, we have six degrees of freedom from the sixth-order boundarylayer system (4.5)-(4.7), two degrees of freedom from the unknown matching functions $f(\tau)$ and $g(\tau)$, and one degree of freedom from half of the second-order interior system (2.1). (By symmetry, the other other degree of freedom in the interior system must be set by the conditions at the other end of tube.) This makes a total of nine degrees of freedom.

We then have three boundary conditions (4.8) on the boundary-layer solution at $z=0$, and two conditions (4.14) on the interior solution at $\check{z}=0$. We therefore require that the remaining conditions (4.11)-(4.13) impose precisely four constraints on the boundary layer as $z \rightarrow \infty$.

By comparison with circular limit described in Appendix $\mathrm{C}$, in addition to the linear behaviour in (4.11)-(4.13), we would expect two modes that grow exponentially in $z$ and two modes that decay in $z$ to make up the six independent solutions of (4.5)-(4.7). The conditions (4.11)-(4.13) define the two linear modes, and also set the coefficients of the two growing modes to zero. Thus they do indeed impose four constraints on the system.

\section{B.2 Boundary-layer eigenvalue problem of $\S 5.2$}

As above, there are six degrees of freedom from the solutions to the boundary-layer system (4.5)-(4.7). In the asymptotic conditions (5.2)-(5.4) there is one unknown function and one unknown eigenvalue. This gives a total of eight degrees of freedom.

The boundary conditions (4.8) at $z=0$ impose three constraints. By the same argument as given in Appendix B.1 above, the asymptotic conditions (4.11)-(4.13) impose four constraints. The final degree 
of freedom is in the undetermined normalisation; the problem is linear and all the boundary conditions are homogeneous. Therefore we have the correct number of boundary conditions for the problem.

\section{Limit of a circular cross-section $\left(\sigma_{0} \rightarrow \infty\right)$}

In this appendix, we consider the boundary-layer system (4.5)-(4.13) in the limit in which the elliptical cross-section of the undeformed tube becomes circular. The equations can be solved using Fourier series in the azimuthal coordinate, and explicit matching conditions obtained to apply to the interior solution.

\section{C.1 Fourier representation}

In the limit $\sigma_{0} \rightarrow \infty$, the undeformed cross-section of the tube becomes circular, and we have

$$
c \sim 2 \mathrm{e}^{-\sigma_{0}}, \quad h(\tau) \rightarrow 1, \quad \bar{B} \rightarrow-1 .
$$

The boundary-layer system (4.5)-(4.7), (4.8), (4.11)-(4.13) then looses any explicit dependence on $\tau$. Since the equations are linear, we expect to find solutions that have sinusoidal behaviour in $\tau$. Taking into account the required $\pi$-periodicity and parity, we seek a solution of the form

$$
\xi=\sum_{n=0}^{\infty} a_{n}(z) \cos (2 n \tau), \quad \eta=\sum_{n=0}^{\infty} b_{n}(z) \sin (2 n \tau), \quad \zeta=\sum_{n=0}^{\infty} c_{n}(z) \cos (2 n \tau) .
$$

\section{C.2 Equations and boundary conditions}

On the substitution of (A.21) into the governing equations (4.5)-(4.7), the different Fourier modes decouple. For each mode $n$, we obtain

$$
\begin{aligned}
& \mathscr{F}\left(1-v^{2}\right) a_{n}^{\prime \prime}-v c_{n}^{\prime}-a_{n}-2 n b_{n}=0, \\
&\left(\frac{1}{2}+\mathscr{F}\left(1-v^{2}\right)\right) b_{n}^{\prime \prime}-n(1+v) c_{n}^{\prime}-2 n a_{n}-4 n^{2} b_{n}=0, \\
&\left(1+2 \mathscr{F}\left(1-v^{2}\right)\right) c_{n}^{\prime \prime}+\left(v+\mathscr{F}\left(1-v^{2}\right)\right) a_{n}^{\prime} \\
&+n\left((1+v)+2 \mathscr{F}\left(1-v^{2}\right)\right) b_{n}^{\prime}-2 n^{2}(1-v) c_{n}=0,
\end{aligned}
$$

where primes denote derivatives with respect to $z$. From (4.8), the boundary conditions at $z=0$ are

$$
a_{n}=0, \quad b_{n}=0, \quad c_{n}=0 \quad \text { at } \quad z=0,
$$

while (4.11)-(4.13) give the far-field conditions

$$
a_{n} \sim-4 n^{2} F_{n}\left(z-z_{n}^{*}\right), \quad b_{n} \sim 2 n F_{n}\left(z-z_{n}^{*}\right), \quad c_{n} \sim F_{n} \quad \text { as } \quad z \rightarrow \infty,
$$

where $F_{n}$ and $z_{n}^{*}$ are arbitrary constants. It turns out that the $F_{n}$ are determined solely by the matching with the interior solution, while the $z_{n}^{*}$ are determined solely by the solution of the boundary-layer system. ${ }^{3}$

\footnotetext{
${ }^{3}$ In general, one might expect both $F_{n}$ and $z_{n}^{*}$ to be involved in the matching condition and influenced by the interior solution. However, since this system is linear, $F_{n}$ simply determines the amplitude of the mode, independent of the value of $z_{n}^{*}$. Hence each $z_{n}^{*}$ is fixed by the boundary-layer system, and is not affected by the matching to the interior solution.
} 


\section{C.3 Boundary-layer solution}

For each $n$, we have set of three coupled linear ODEs with constant coefficients. Overall each system is sixth order in $z$, with one free parameter $z_{n}^{*}$. (We regard the $F_{n}$ as external parameters, imposed by the matching.) We therefore need seven boundary conditions.

We find that the system (A.22)-(A.24) has four linearly independent exponential solutions, two of which grow as $z \rightarrow \infty$ and two of which decay. There is also a general linear $(A+B z)$ component, making up the remaining two solutions. The boundary conditions (A.25) at $z=0$ impose three constraints. The conditions (A.26) as $z \rightarrow \infty$ require the coefficients of the two growing exponentials to be zero, and also set the two coefficients of the linear component; making a total of four constraints.

These conditions thus give the required seven constraints, and we can write down an explicit analytic solution for $a_{n}(z), b_{n}(z), c_{n}(z)$. Each of the functions takes the form

$$
A+B z+C \mathrm{e}^{-\kappa_{1} z}+D \mathrm{e}^{-\kappa_{2} z}
$$

However, the coefficients $\{A, B, C, D\}$ and the decay rates $\left\{\kappa_{1}, \kappa_{2}\right\}$ are complicated functions of $\mathscr{F}, v$ and $n$, so in the interests of brevity we omit the full expressions here.

From these solutions we can obtain an expression for the offset $z_{n}^{*}$ for each Fourier mode. The offset also depends on $\mathscr{F}$ and $v$. Some representative values are plotted in figure A.7. For $\mathscr{F} \ll 1$ we find the asymptotic behaviour

$$
z_{n}^{*} \sim k_{n} \mathscr{F}^{-1 / 2}
$$

where

$$
k_{1}=\frac{\sqrt{5}}{10}, \quad k_{2}=\frac{\sqrt{17}}{68}, \quad k_{3}=\frac{\sqrt{37}}{222}, \quad k_{4}=\frac{\sqrt{65}}{520},
$$

independent of $v$. For $\mathscr{F} \gg 1$, we have $z_{n}^{*} \sim C_{n}(v) \mathscr{F}^{-1 / 2}$, with the same power of $\mathscr{F}$, but the coefficients are now complicated functions of $v$. Plots of $z_{n}^{*}$ as a function of $\mathscr{F}$ are show in figure A.7.

Given the Fourier coefficients of the displacements $(\xi, \eta, \zeta)$ we can use (3.19)-(3.21) to compute the corresponding coefficients for the stresses $(\tilde{N}, \tilde{S}, \tilde{\Sigma})$. Writing

$$
\tilde{N}=\sum_{n=0}^{\infty} \alpha_{n}(z) \cos (2 n \tau), \quad \tilde{S}=\sum_{n=0}^{\infty} \beta_{n}(z) \sin (2 n \tau), \quad \tilde{\Sigma}=\sum_{n=0}^{\infty} \gamma_{n}(z) \cos (2 n \tau),
$$

we find that

$$
\begin{aligned}
& \alpha_{n}(z)=12\left(a_{n}+2 n b_{n}+v c_{n}^{\prime}\right), \\
& \beta_{n}(z)=6(1-v)\left(b_{n}^{\prime}-2 n c_{n}\right), \\
& \gamma_{n}(z)=12\left(c_{n}^{\prime}+v a_{n}+2 n v b_{n}\right) .
\end{aligned}
$$

Plots of the displacement and stress amplitudes for the $n=1$ mode at a relatively small value of $\mathscr{F}$ are shown in figure A.8. We observe the linear behaviour of the displacements and the decay of the stresses as $z \rightarrow \infty$. We also note the appearance of two distinct length scales in $z$. For both the displacements and the stresses, there appears to be an inner layer occupying $0 \leqslant z \lesssim 0.3$ and an outer layer occupying $z \gtrsim 0.3$. (These layers, which appear for general $\sigma_{0}$ when $\mathscr{F} \ll 1$, are discussed in more detail in $\S 6.6$.) 


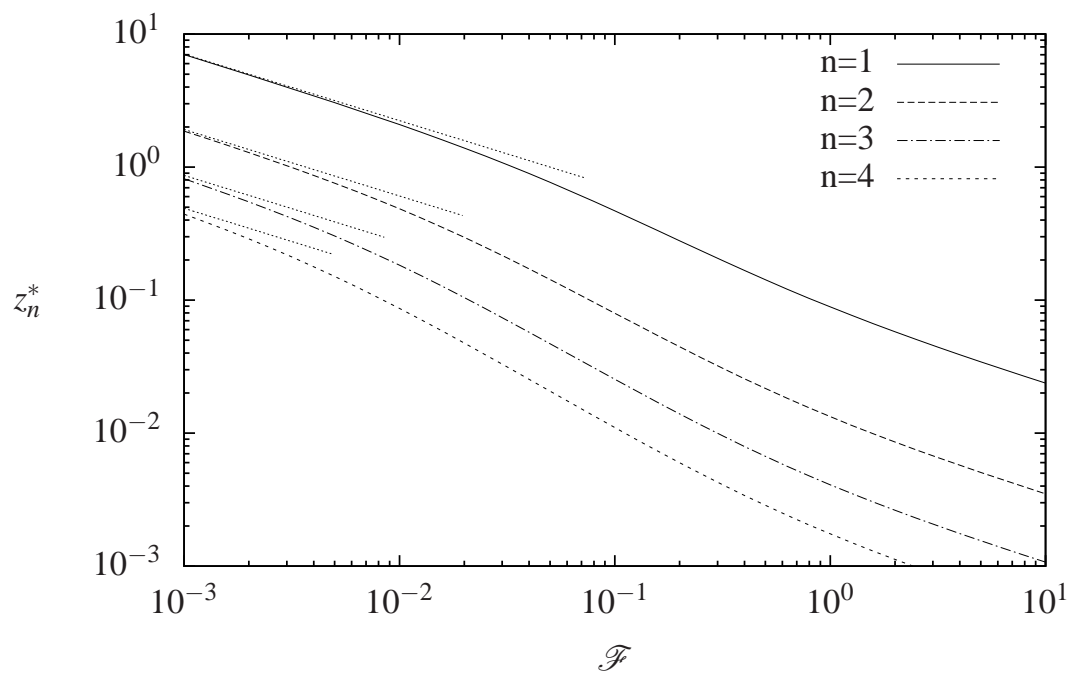

FIG. A.7. The offset $z_{n}^{*}$ as a function of the scaled axial tension $\mathscr{F}$ for the case of a circular cross-section with $v=0.49$. The dotted lines to the left show the asymptotic behaviour $z_{n}^{*} \sim k_{n} \mathscr{F}^{-1 / 2}$ for $\mathscr{F} \ll 1$, with the $k_{n}$ given by (A.29).

\section{C.4 Matching condition imposed on the interior solution}

The boundary conditions imposed on the interior solution are that as $\breve{z} \rightarrow 0, \check{\eta}$ matches the asymptotic form of $\eta$ as $z \rightarrow \infty$. Expressing (A.26), in terms of the behaviour (4.12)-(4.13), we find

$$
f(\tau)=\sum_{n=1}^{\infty} F_{n} \cos (2 n \tau), \quad g(\tau)=-2 \sum_{n=1}^{\infty} n F_{n} z_{n}^{*} \sin (2 n \tau) .
$$

The matching conditions (4.14) then yield

$$
\check{\eta}=-\frac{2}{\ell} \sum_{n=1}^{\infty} n F_{n} z_{n}^{*} \sin (2 n \tau) \quad \text { and } \quad \frac{\partial \check{\eta}}{\partial \check{z}}=2 \sum_{n=1}^{\infty} n F_{n} \sin (2 n \tau) \quad \text { at } \quad \check{z}=0 \text {, }
$$

for some set of constants $F_{n}$. Alternatively, we can use the orthogonality of the Fourier modes to eliminate the $F_{n}$ from (A.35). Doing so, we obtain a set of homogeneous integral constraints, indexed by $n$ :

$$
\int_{0}^{2 \pi}\left(\frac{z_{n}^{*}}{\ell} \frac{\partial \check{\eta}}{\partial \check{z}}+\check{\eta}\right) \sin (2 n \tau) \mathrm{d} \tau=0 \quad \text { at } \quad \check{z}=0
$$

\section{Reformulation in terms of stress variables}

Motivated by the underlying physics, we consider a change of variables from the displacement-based $(\xi, \eta, \zeta)$ to use variables corresponding to the three in-plane stress components in the shell: the azimuthal stress $\tilde{N}$, the axial stress $\tilde{\Sigma}$, and shear stress $\tilde{S}$. These stress variables are given in terms of $(\xi, \eta, \zeta)$ by (3.19)-(3.21).

By manipulating (3.19)-(3.21) and using the boundary conditions (4.8) at $z=0$, we derive in turn 
(a)

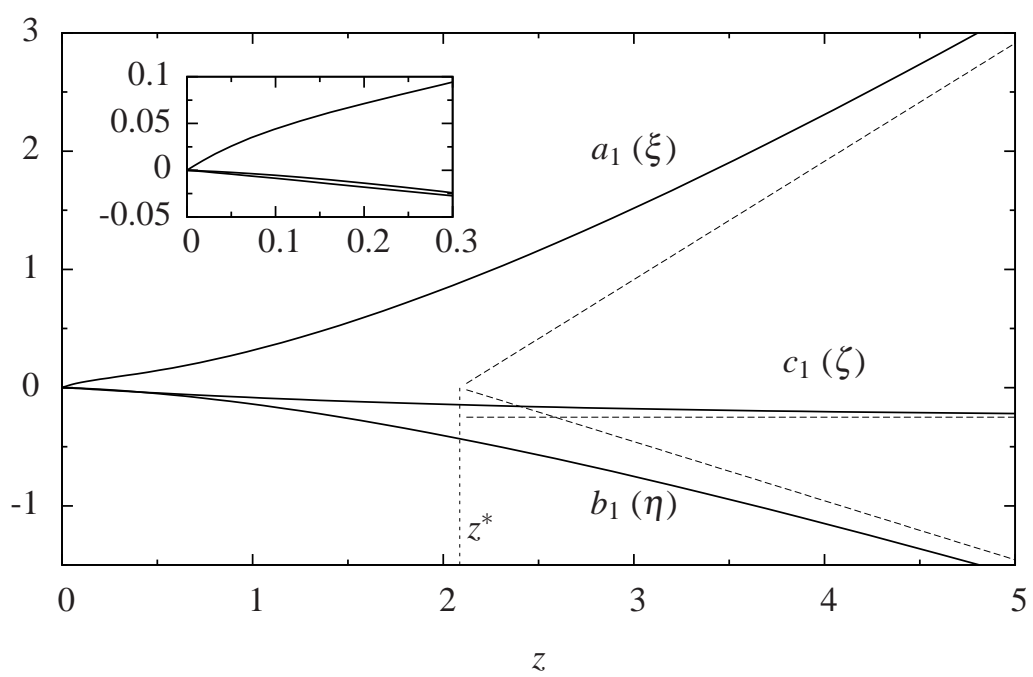

(b)

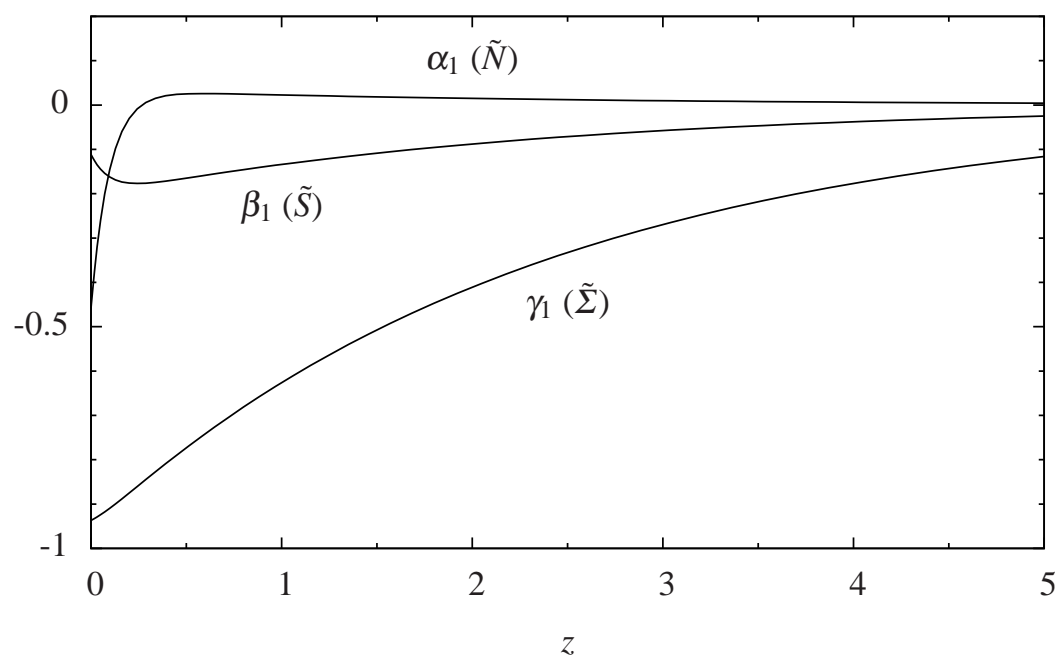

FIG. A.8. Analytical solutions for the $n=1$ mode with $\mathscr{F}=0.01$ and $v=0.49$ in the limit of a circular cross-section. (a) The Fourier coefficients $a_{1}, b_{1}, c_{1}$ in the expansions (A.21) for the displacements $\xi, \eta, \zeta$. The inset shows the behaviour near $z=0$ in more detail. The dashed lines show the asymptotic behaviour as $z \rightarrow \infty$. Note that the linear asymptotes for $a_{1}$ and $b_{1}$ both meet the $z$ axis at the same point $z=z^{*}$. (b) The Fourier coefficients $\alpha_{1}, \beta_{1}, \gamma_{1}$ in the expansions (A.30) for the stresses $\tilde{N}, \tilde{S}, \tilde{\Sigma}$. 
the following expressions for the displacement functions in terms of the stresses:

$$
\begin{aligned}
& \zeta(\tau, z)=\frac{1}{12\left(1-v^{2}\right)} \int_{0}^{z}\left(\tilde{\Sigma}\left(\tau, z^{\prime}\right)-v \tilde{N}\left(\tau, z^{\prime}\right)\right) \mathrm{d} z^{\prime} \\
& \eta(\tau, z)=\int_{0}^{z}\left(\frac{2 h(\tau) \tilde{S}\left(\tau, z^{\prime}\right)}{12(1-v)}-\frac{\partial \zeta\left(\tau, z^{\prime}\right)}{\partial \tau}\right) \mathrm{d} z^{\prime} \\
& \xi(\tau, z)=-\frac{h(\tau)}{\bar{B}(\tau)}\left(\frac{\tilde{N}(\tau, z)-v \tilde{\Sigma}(\tau, z)}{12\left(1-v^{2}\right)}-\frac{1}{h(\tau)} \frac{\partial}{\partial \tau}\left(\frac{\eta(\tau, z)}{h(\tau)}\right)\right) .
\end{aligned}
$$

Using (A.37)-(A.39), we then obtain

$$
\begin{aligned}
12 \frac{\partial \zeta}{\partial z} & =\frac{\tilde{\Sigma}-v \tilde{N}}{1-v^{2}} \\
12 \frac{\partial^{2}}{\partial z^{2}}\left(\frac{\eta}{h}\right) & =\frac{2}{(1-v)} \frac{\partial \tilde{S}}{\partial z}-\frac{1}{h} \frac{\partial}{\partial \tau}\left(\frac{\tilde{\Sigma}-v \tilde{N}}{1-v^{2}}\right), \\
12 \bar{B} \frac{\partial^{2}}{\partial z^{2}}\left(\frac{\xi}{h}\right) & =-\frac{\partial^{2}}{\partial z^{2}}\left(\frac{\tilde{N}-v \tilde{\Sigma}}{1-v^{2}}\right)+\frac{2}{(1-v) h} \frac{\partial^{2} \tilde{S}}{\partial \tau \partial z} \\
12\left(-\frac{1}{h} \frac{\partial}{\partial \tau}\left(\frac{1}{h} \frac{\partial}{\partial \tau}\left(\frac{\tilde{\Sigma}-v \tilde{N}}{1-v^{2}}\right)\right),\right. & \left.\tilde{N}-\frac{1}{h} \frac{\partial}{\partial \tau}\left(\frac{\eta}{h}\right)\right)=\frac{v^{2}}{1-v^{2}}
\end{aligned}
$$

These expressions can be used to eliminate $\xi, \eta$ and $\zeta$ from the governing equations (4.1)-(4.3). We thus obtain the new governing equations

$$
\begin{aligned}
\bar{B}^{2} \tilde{N}+\mathscr{F}\left(-\tilde{N}_{z z}+v \tilde{\Sigma}_{z z}+2(1+v) \tilde{S}_{\tau z}-\tilde{\Sigma}_{\tau \tau}+v \tilde{N}_{\tau \tau}\right) & =0, \\
\tilde{N}_{\tau}+\tilde{S}_{z}+\mathscr{F}\left(2(1+v) \tilde{S}_{z}-\tilde{\Sigma}_{\tau}+v \tilde{N}_{\tau}\right) & =0, \\
\tilde{S}_{\tau}+\tilde{\Sigma}_{z}+\mathscr{F}\left((1-2 v) \tilde{N}_{z}+(2-v) \tilde{\Sigma}_{z}\right) & =0,
\end{aligned}
$$

where a subscript $z$ represents the partial derivative with respect to $z$, but a subscript $\tau$ represents the operator

$$
\frac{1}{h(\tau)} \frac{\partial}{\partial \tau}
$$

The system (A.44)-(A.46) is 4th order in $z$ and 4th order in $\tau$. The two orders in $z$ lost from the original system (4.5)-(4.7) are accounted for in the two integrals that appear in the recovery equations (A.37)(A.39).

The boundary conditions on $\tilde{N}, \tilde{\Sigma}$ and $\tilde{S}$ come from the conditions (4.8) and (4.11)-(4.13) on $\xi, \eta$, and $\zeta$. We must still have periodicity in $\tau$. At $z=0$, (A.37) and (A.38) give $\zeta=\eta=0$ automatically. Equation (A.39) then implies

$$
\tilde{N}-v \tilde{\Sigma}=0 \quad \text { on } \quad z=0 .
$$

As $z \rightarrow \infty,(4.11)-(4.13)$ imply that the stresses must decay, so

$$
\tilde{N}, \tilde{\Sigma}, \tilde{S} \rightarrow 0 \quad \text { as } \quad z \rightarrow \infty .
$$


These conditions equate to only two constraints, since if $\tilde{N}, \Sigma \rightarrow 0$ then (A.46) and periodicity imply that $\tilde{S} \rightarrow 0$ also.

Inserting (A.37) and (A.38) into (4.13) and then (4.12) we obtain two integral equations to determine the matching functions $f$ and $g$ :

$$
\begin{aligned}
f(\tau) & =\frac{1}{12\left(1-v^{2}\right)} \int_{0}^{\infty} \tilde{\Sigma}(z, \tau)-v \tilde{N}(z, \tau) \mathrm{d} z, \\
g(\tau)= & \frac{1}{12\left(1-v^{2}\right)} \frac{\mathrm{d}}{\mathrm{d} \tau}\left(\int_{0}^{\infty} \int_{z}^{\infty} \tilde{\Sigma}\left(z^{\prime}, \tau\right)-v \tilde{N}\left(z^{\prime}, \tau\right) \mathrm{d} z^{\prime} \mathrm{d} z\right) \\
& \quad \frac{1}{6(1-v)} \int_{0}^{\infty} h(\tau) \tilde{S}(z, \tau) \mathrm{d} z .
\end{aligned}
$$

